\title{
Well-defined biomimetic surfaces to characterize glycosaminoglycan- mediated interactions on the molecular, supramolecular and cellular levels
}

\author{
Elisa Migliorini ${ }^{\text {a, b, } 1}$, Dhruv Thakar ${ }^{\text {a, b, } 1}$, Rabia Sadir ${ }^{\text {c, d, e }}$, Tino Pleiner ${ }^{\mathrm{f}}$, \\ Françoise Baleux $^{g}$, Hugues Lortat-Jacob ${ }^{\text {c, d, e }}$, Liliane Coche-Guerente ${ }^{\mathrm{a}, \mathrm{b}}$, \\ Ralf P. Richter ${ }^{\text {a, b, h, i, * }}$ \\ a Univ. Grenoble Alpes, Departement de Chimie Moléculaire (DCM), Grenoble, France \\ ${ }^{\mathrm{b}}$ CNRS, DCM, Grenoble, France \\ ${ }^{\mathrm{c}}$ Univ. Grenoble Alpes, Institut de Biologie Structurale (IBS), Grenoble, France \\ d CNRS, IBS, Grenoble, France \\ e CEA, DSV, IBS, Grenoble, France \\ ${ }^{\mathrm{f}}$ Department of Cellular Logistics, Max Planck Institute for Biophysical Chemistry, Göttingen, Germany \\ ${ }^{g}$ Unité de Chimie des Biomolécules, Département de Biologie Structurale et Chimie, Institut Pasteur, Paris, France \\ h CIC biomaGUNE, Biosurfaces Unit, San Sebastian, Spain \\ i Max Planck Institute for Intelligent Systems, Stuttgart, Germany
}

\section{A R T I C L E I N F O}

\section{Article history:}

Received 21 May 2014

Accepted 10 July 2014

Available online 1 August 2014

\section{Keywords:}

Glycosaminoglycan

Chemokine

T-lymphocyte adhesion

Quartz crystal microbalance

Spectroscopic ellipsometry

Surface plasmon resonance

\begin{abstract}
A B S T R A C T
Glycosaminoglycans (GAGs) are ubiquitously present at the cell surface and in extracellular matrix, and crucial for matrix assembly, cell-cell and cell-matrix interactions. The supramolecular presentation of GAG chains, along with other matrix components, is likely to be functionally important but remains challenging to control and to characterize, both in vivo and in vitro. We present a method to create welldefined biomimetic surfaces that display GAGs, either alone or together with other cell ligands, in a background that suppresses non-specific binding. Through the design of the immobilization platform - a streptavidin monolayer serves as a molecular breadboard for the attachment of various biotinylated ligands - and a set of surface-sensitive in situ analysis techniques (including quartz crystal microbalance and spectroscopic ellipsometry), the biomimetic surfaces are tailor made with tight control on biomolecular orientation, surface density and lateral mobility. Analysing the interactions between a selected GAG (heparan sulphate, HS) and the HS-binding chemokine CXCL12 $\alpha$ (also called SDF- $1 \alpha$ ), we demonstrate that these surfaces are versatile for biomolecular and cellular interaction studies. T-lymphocytes are found to adhere specifically to surfaces presenting CXCL12 $\alpha$, both when reversibly bound through HS and when irreversibly immobilized on the inert surface, even in the absence of any bona fide cell adhesion ligand. Moreover, surfaces which present both HS-bound CXCL12 $\alpha$ and the intercellular adhesion molecule 1 (ICAM-1) synergistically promote cell adhesion. Our surface biofunctionalization strategy should be broadly applicable for functional studies that require a well-defined supramolecular presentation of GAGs along with other matrix or cell-surface components.
\end{abstract}

๑) 2014 Elsevier Ltd. All rights reserved.

\footnotetext{
* Corresponding author. Biosurfaces Unit, CIC biomaGUNE, Paseo Miramon 182, 20009 Donostia-San Sebastian, Spain. Tel.: +34 9430053 29; fax: +34943005315.

E-mail address: rrichter@cicbiomagune.es (R.P. Richter).

1 E.M. and D.T. contributed equally to this work.
}

\section{Introduction}

Glycosaminoglycans (GAGs), a group of acidic and linear polysaccharides including e.g. heparan sulphates (HS) and hyaluronan (HA), are ubiquitously present at the cell surface and in extracellular matrix. They interact with many structural (e.g. collagen, fibronectin) and signalling (e.g. chemokines, growth factors) proteins and thereby regulate matrix assembly and remodelling, as well as cell-matrix and cell-cell interactions [1]. Typically, GAG 
function relies on the integration of multiple interactions rather than on individual binding events. Examples are (i) the remodelling of extracellular matrices by GAGs binding to structural proteins $[2,3]$, by GAG-crosslinking proteins $[4,5]$ or by bulky GAG-binding proteoglycans $[6-8]$, (ii) the sequestration $[9,10]$ and tightly regulated mobility [11] of chemokines or growth factors in matrix and (iii) the presentation of chemokines at controlled densities or in the form of gradients to promote distinct cellular responses such as adhesion or directed migration [12,13].

In all these cases, the local arrangement and density of GAGs, along with other cell surface or matrix components, is of key functional importance. Studies in this direction, however, are challenging because the supramolecular presentation of GAG chains is difficult to control and to characterize, not only in vivo but also in vitro. For example, GAGs have so far been largely neglected in the design of in vitro cell migration assays [14], despite their recognized functional importance. A likely reason is the limited commercial availability of sufficiently pure and suitably functionalized GAGs, and of methodologies to integrate GAGs into assemblies that mimic the presentation at the cell surface or in matrix well. Only few studies demonstrate, so far, the possibility to immobilize and to control GAG surface densities and/or orientations on supports [15-18]. Moreover, to the best of our knowledge the cellular interaction with such surfaces has not been studied. To study the role of GAG-protein interactions in matrix assembly and in cell behaviour in vitro, it would be desirable to be able to prepare materials that present GAGs together with other relevant biomolecules in such a way that the orientation, density and lateral mobility of the exposed biomolecules can be controlled and tuned.

Here, we present a method to fabricate biomimetic surfaces that display GAGs and other biomolecules of interest at well-defined orientation, density and lateral mobility (Fig. 1). The method relies on a stratified monolayer of streptavidin (SAv) and selfassembly through strong and specific interactions. Embedded in a background that is largely inert to the undesired nonspecific adhesion of biomolecules or cells, the SAv monolayer serves as a molecular breadboard for the selective attachment of biotinylated molecules. The method is generic in the sense that various biomolecules can be (co-) immobilized at tuneable surface densities, either through a site-specifically attached biotin tag, or if that is not available, through biotinylated adapter molecules. In particular, GAGs are immobilized with controlled orientation through a biotin tag introduced at the reducing end. As a prerequisite of the present work, a method for the preparation of stable and terminally functionalized GAG conjugates was recently developed (Thakar D, Migliorini E, Guerente L, Sadir R, Lortat-Jacob H, Boturyn D, Renaudet O, Labbé P, Richter, RP manuscript submitted).

Two in situ surface sensitive analytical techniques, quartz crystal microbalance (QCM-D) and spectroscopic ellipsometry (SE), were combined to monitor in real time the assembly of the biomimetic surfaces and to ascertain that the desired functionalities are indeed realized. In particular, QCM-D provides time-resolved information about the assembly process, including overall film morphology and mechanics [19], while SE enables time-resolved and label-free quantification of biomolecular surface densities and binding stoichiometries [20].

To demonstrate the versatility of our approach for wellcontrolled and quantitative biomolecular and cellular studies, we selected HS as GAG, and stromal-cell derived factor 1 (CXCL12 $\alpha$, also called SDF-1 $\alpha$ ) as GAG-binding chemokine [21]. We first quantify the kinetics of CXCL12 $\alpha$ binding to HS-displaying surfaces by surface plasmon resonance and then investigate the adhesion of Jurkat cells as a CXCL12 $\alpha$ sensitive T-lymphocyte cell line [13] to a range of biomimetic surfaces, including surfaces that present HSbound CXCL12 $\alpha$ along with the intercellular adhesion molecule 1 (ICAM-1). CXCL12 $\alpha$ is known to bind with high affinity to the T cellsurface receptor CXCR4 [22,23]; the ensuing activation of T cells as well as the binding of T cells to ICAM- 1 at the endothelial cell

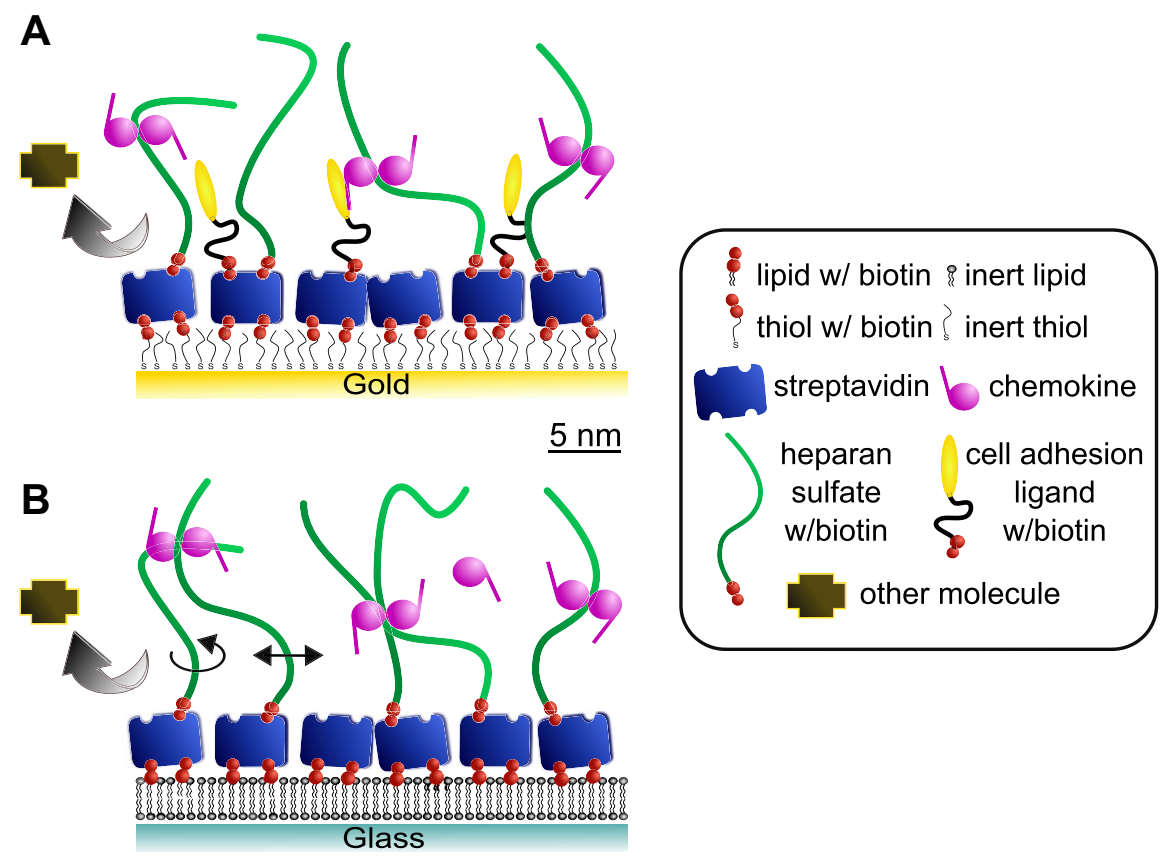

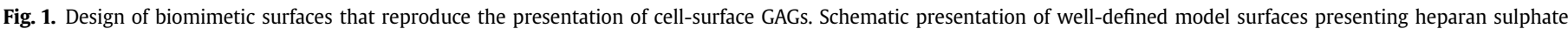

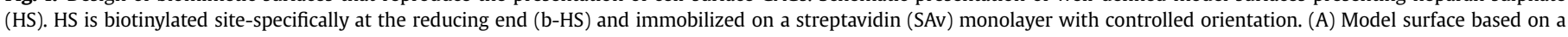

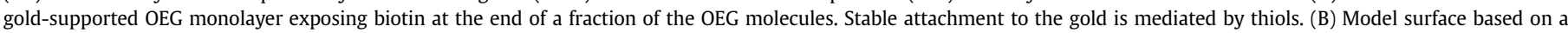

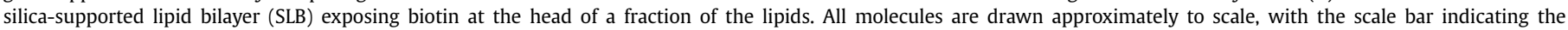

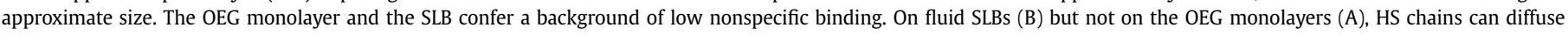
laterally together with the anchoring SAv (thin arrows). 
surface of blood vessels are of particular importance in immune cell trafficking [12,24,25]. With this study, surfaces thus become available that mimic selected aspects of the endothelial cell surface, towards mechanistic cellular studies in an environment that is well-defined and tuneable.

\section{Methods}

\subsection{Buffer, heparan sulphate and proteins}

The working buffer used for all experiments and for protein dilution was made of $10 \mathrm{~mm}$ Hepes (Fisher, Illkirch, France) at pH 7.4 and $150 \mathrm{~mm} \mathrm{NaCl}$ (Sigma Aldrich, Saint-Quentin Fallavier, France) in ultrapure water.

Heparan sulphate (HS) derived from porcine intestinal mucosa with an average molecular weight of $12 \mathrm{kDa}$ and a polydispersity of 1.59 [26] (Celsus Laboratories, Cincinnati, OH, USA) was conjugated with biotin through an oligoethylene glycol linker of approximately $\sim 1 \mathrm{~nm}$ length, site-specifically attached to the reducing end by oxime ligation. In contrast to the conventionally used hydrazone ligation, oxime ligation produces conjugates that are stable for many weeks in aqueous solution

CXCL12 $\alpha$ (amino acids 1 to $68 ; 8.1 \mathrm{kDa}$ ) was produced by solid-phase peptide synthesis as previously reported [9]. The same CXCL12 $\alpha$ construct with a biotin conjugated to the C-terminal lysine through a tetraethylene glycol (OEG) linker (bCXCL12 $\alpha ; 8.6 \mathrm{kDa}$ ), was also produced by solid-phase peptide synthesis. A protein construct containing two Z-domains of protein A, separated by an extended peptide spacer from an Avi-tag carrying a single biotin (b-ZZ, $31.8 \mathrm{kDa}$ ) was produced recombinantly in Escherichia coli by co-expression with the biotin ligase BirA Lyophilized streptavidin (SAv, $60 \mathrm{kDa}$ ), bovine serum albumin (BSA) and fibronectin (Fn) were purchased from Sigma Aldrich. A chimera of the Fc part of IgG and two human ICAM-1 extracellular domains (Fc-ICAM-1; $152 \mathrm{kDa}$ ) was obtained from R\&D Systems (Minneapolis, MN, USA). All proteins were diluted to concentrations between 0.2 and $1 \mathrm{mg} / \mathrm{mL}$ in autoclaved working buffer and stored at $-20^{\circ} \mathrm{C}$. Thawed protein solutions were used within 5 days and further diluted as desired.

\subsection{Sensors and surface preparation}

QCM-D sensors with gold (QSX301) and silica (QSX303) coatings were purchased from Biolin Scientific (Västra Frölunda, Sweden). Appropriately sized silicon wafers with a native oxide layer of less than $2 \mathrm{~nm}$ thickness or with an optically opaque gold coating (100 nm, sputter-coated) were used for SE measurements. SPR gold-coated sensor chips (SIA Kit Au) were purchased from Biacore (GE Healthcare Bio-Sciences, Uppsala, Sweden). Glass cover slips $\left(24 \times 24 \mathrm{~mm}^{2}\right.$; Menzel Gläser Braunschweig, Germany) for cellular studies were cleaned by immersion in freshly prepared piranha solution (i.e. a 1:3 $(v / v)$ mixture of $\mathrm{H}_{2} \mathrm{O}_{2}$ (ACROS Organics, New Jersey, USA) and concentrated $\mathrm{H}_{2} \mathrm{SO}_{4}$ (Sigma Aldrich)) for $1 \mathrm{~h}$, rinsing with ultrapure water, and blow-drying with $\mathrm{N}_{2}$. They were used as such or sputter-coated with a titanium adhesion layer $(\sim 0.5 \mathrm{~nm})$ and a semi-transparent gold film $(\sim 5 \mathrm{~nm})$. Al substrates were exposed to UV/ozone (Jelight, Irvine, CA, USA) for $10 \mathrm{~min}$ prior to further use.

\subsubsection{Functionalization of surfaces with a biotin-displaying and otherwise inert background}

Gold-coated surfaces were immersed overnight in an ethanolic solution (Fisher Illkirch, France) of OEG disulfide and biotinylated OEG thiol (Polypure, Oslo, Norway) at a total concentration of $1 \mathrm{~mm}$ and a molar ratio of thiol equivalents of 999:1, and subsequently for $20 \mathrm{~min}$ in a stirred solution of pure ethanol, and blow-dried in $\mathrm{N}_{2}$ Biotin-functionalized supported lipid bilayers (SLBs) were prepared by the method of vesicle spreading though exposure of small unilamellar vesicles (SUVs; at $50 \mu \mathrm{g}$ / $\mathrm{mL}$ in working buffer supplemented with $2 \mathrm{~mm} \mathrm{CaCl}_{2}$ (VWR International, Leuven, Belgium)) to silica-coated surfaces, as described earlier [27]. SUVs were prepared by sonication, as described earlier [28], from mixtures of dioleoylphosphatidylcholine (DOPC) and dioleoylphosphatidylethanolamine-CAP-biotin (DOPE-CAP-b) (Avant Polar lipids, Alabaster, AL, USA) in a molar ratio of 95:5.

\subsubsection{Surface functionalization with $B S A$ and $F n$}

BSA and Fn were physisorbed on uncoated glass cover slips. To this end, the cover slips were exposed to solutions of either BSA at $5 \mathrm{mg} / \mathrm{mL}$ or Fn at $5 \mu \mathrm{g} / \mathrm{mL}$ in working buffer for $20 \mathrm{~min}$.

\subsection{Assembly of biomimetic surface coatings}

The rationale behind the design of the self-organized biomolecular assemblies is provided in the results section (Fig. 1). Unless indicated otherwise, the following concentrations and exposure times were used: SAv - $20 \mu \mathrm{g} / \mathrm{mL}, 20 \mathrm{~min}$; b-HS $50 \mu \mathrm{g} / \mathrm{mL}, 10 \mathrm{~min}$; CXCL12 $\alpha-5 \mu \mathrm{g} / \mathrm{mL}, 20 \mathrm{~min}$; Fc-ICAM- $1-0.1 \mu \mathrm{m}, 30 \mathrm{~min}$. Under these conditions, binding is expected to saturate or equilibrate, irrespective of whether the solution is flown (in QCM-D and SPR measurements), stirred (in SE measurements) or still (for cell assays). In QCM-D measurements, the injection was sometimes also stopped earlier once the binding curve had reached a plateau. To obtain surfaces that display HS-bound CXCL12 $\alpha$ together with Fc-ICAM-1, b-HS $(1 \mu \mathrm{g} / \mathrm{mL} ; 30 \mathrm{~min})$ and $\mathrm{b}-\mathrm{ZZ}(0.05 \mu \mathrm{m} ; 5 \mathrm{~min})$ were sequentially exposed to the $\mathrm{SAv}$ coated surfaces. Here, the concentrations and incubation times were chosen to obtain desired surface densities (see Fig. S4 for details). Fc-ICAM-1 and CXCL12 $\alpha$ were then incubated until saturation and equilibrium, respectively, were reached.

For QCM-D measurements, exposure to $20 \mu \mathrm{g} / \mathrm{mL}$ SAv was routinely preceded by a first SAv injection at low concentration $(1 \mu \mathrm{g} / \mathrm{mL})$ and decreased flow rate $(6 \mu \mathrm{L} /$ $\mathrm{min}$ ) for $5 \mathrm{~min}$, to confirm the absence of protein depletion resulting from undesired adsorption to the walls of the tubing or the QCM-D flow module due to insufficient passivation: any depletion of SAv from the solution would result in a decreased binding rate (compared to the established standard rate of $-0.5 \pm 0.1 \mathrm{~Hz} / \mathrm{min}$, see Fig. 2A-B).

\subsection{Quartz crystal microbalance with dissipation monitoring (QCM-D)}

QCM-D measures the changes in resonance frequency, $\Delta f$, and dissipation, $\Delta D$, of a sensor crystal upon molecular adsorption on its surface. The QCM-D response is sensitive to the mass (including hydrodynamically coupled water) and the mechanical properties of the surface-bound layer. Measurements were performed with a Q-Sense E4 system equipped with 4 independent Flow Modules (Biolin Scientific). Sensor functionalization with biotinylated OEG monolayers was performed ex situ before the measurement. All other surface functionalization steps proceeded in situ. The system was operated in flow mode with a flow rate of typically $10 \mu \mathrm{L} / \mathrm{min}$ using a peristaltic pump (ISM935C, Ismatec, Zurich, Switzerland). The working temperature was $24^{\circ} \mathrm{C}$. $\Delta f$ and $\Delta D$ were measured at six overtones $(i=3,5, \ldots, 13)$, corresponding to resonance frequencies of $f_{i} \approx 5,15,25, \ldots, 65 \mathrm{MHz}$; changes in dissipation and normalized frequency, $\Delta f=\Delta f_{i} / i$, of the third overtone $(i=3)$ are presented; any other overtone would have provided comparable information.

For sufficiently rigid biomolecular layers at high surface coverage, the film thickness was estimated from $d=-C / \rho \times \Delta f$, where $\rho$ is the film density and $C=18.06 \mathrm{ng} / \mathrm{cm}^{2} / \mathrm{Hz}$ the mass sensitivity constant for a sensor with a fundamental resonance frequency of $4.95 \mathrm{MHz}$ [19]. For very soft films, such as HS monolayers, this equation is not valid. Here, film thickness was determined by fitting the QCM-D data to a continuum viscoelastic model [29] with the software QTM (D. Johannsmann, Technical University of Clausthal, Germany) [30,31] as described in detail elsewhere [32]. These thickness values are provided as average \pm standard deviation from at least three independent experiments.

\subsection{Spectroscopic ellipsometry (SE)}

SE measures changes in the polarization of light upon reflection at a planar surface. SE was employed in situ with a M2000V system (J. A. Woollam, Lincoln, NE, USA) to quantify the surface density of adsorbed biomolecules in a time-resolved manner [28]. The desired substrate was installed in a custom-built open cuvette featuring a magnetic stirrer for continuous homogenization of the sample solution ( $\sim 150 \mu \mathrm{l}$; samples were pipetted into the solution) and a flow-through system for rapid solution exchange during rinsing steps. Before use, the cuvette walls were passivated against biomolecular binding with BSA [4]. Substrate functionalization with biotinylated OEG monolayers was performed ex situ, before the measurement. All other surface functionalization steps proceeded in situ. Measurements were performed at room temperature.

Surface densities were quantified through fitting of the data to optical models, as described in detail elsewhere $[28,33]$. The opaque metal film and the OEG monolayer on gold-coated silicon wafers were treated as a single isotropic layer and fitted as a B-spline substrate. The bulk silica of native wafers was also modelled as a B-spline substrate. The native oxide film, alone or together with a deposited SLB, were modelled as a single transparent Cauchy layer. Areal mass densities were determined through de Fejter's equation [20], using refractive index increments, $\mathrm{d} n / \mathrm{d} c$, of $0.15 \mathrm{~cm}^{3} / \mathrm{g}$ for b-HS, $0.18 \mathrm{~cm}^{3} / \mathrm{g}$ for all proteins and $0.17 \mathrm{~cm}^{3} / \mathrm{g}$ for lipids.

\subsection{Surface plasmon resonance (SPR)}

SPR measurements were performed with a Biacore T200 (GE Healthcare BioSciences) operated with Biacore T200 evaluation software (version 2.0.1). All measurements were performed at $25^{\circ} \mathrm{C}$ using a working buffer solution supplemented with $0.005 \%(w / w)$ surfactant P20 (an additive employed to reduce nonspecific adsorption to flow system surfaces). The gold-covered Biacore sensor chips were first coated ex situ with a biotinylated OEG monolayer, and further functionalization with SAv and b-HS to saturation proceeded in situ. Binding experiments were performed by injecting CXCL12 $\alpha$ at desired concentrations and at a rate of $75 \mu \mathrm{L} / \mathrm{min}$ for $4.5 \mathrm{~min}$. Between binding assays, the surface was regenerated by two steps of exposure to $2 \mathrm{~m} \mathrm{NaCl}$ for 2 min each. Upon $\mathrm{NaCl}$ treatment, the SPR signal returned to within $5 \mathrm{RU}$ to the level before incubation with chemokine, indicating full regeneration. Reference measurements were performed in parallel on SAv monolayers lacking HS. To obtain the sensorgram shown in Fig. 4A, the reference data were subtracted from the binding curves on HS films. The responses in the reference channel were always below $10 \%$ of the total response, indicating that non-specific binding and solution effects on the SPR response were minor.

\subsection{T-lymphocyte culture and adhesion assays}

The Jurkat cell line was obtained from the European Collection of Cell Cultures (ECACC). Cells were maintained in Roswell Park Memorial Institute (RPMI 1640) 

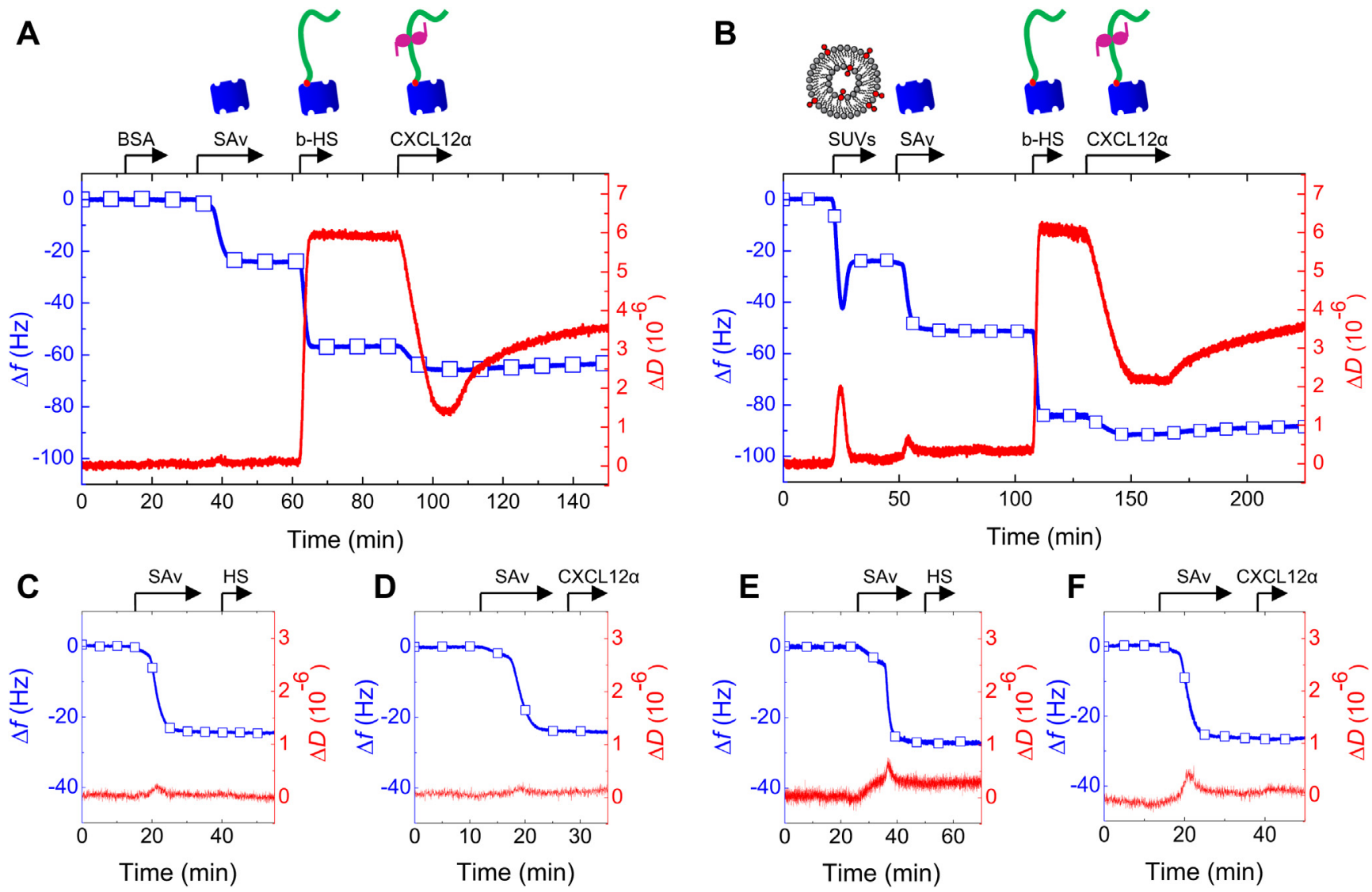

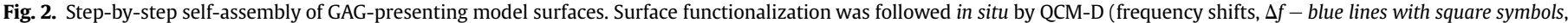

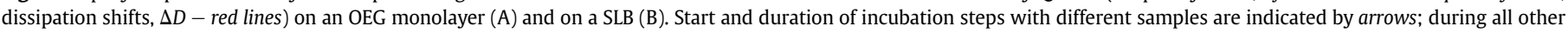

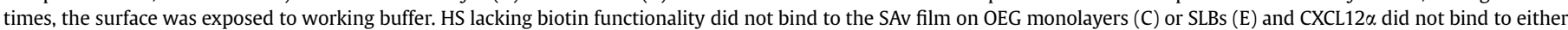
SAv film in the absence of HS (D, F). (For interpretation of the references to colour in this figure legend, the reader is referred to the web version of this article.)

medium, supplemented with $10 \%$ fetal calf serum, 2 mm L-glutamine, 100 units $/ \mathrm{ml}$ penicillin, $100 \mu \mathrm{g} / \mathrm{mL}$ streptomycin (all from LifeTechnologies, Carlsbad, CA, USA). Cells were grown at $37{ }^{\circ} \mathrm{C}$ under a $5 \% \mathrm{CO}_{2}$ atmosphere, and passed at densities between $2 \times 10^{5}$ and $2 \times 10^{6}$ cells $/ \mathrm{mL}$.

For cell adhesion assays, glass coverslips - either uncoated or coated with gold and a biotinylated OEG monolayer - were attached, using a bi-component glue (Picodent, Wipperfürth, Germany), to a custom-built teflon holder, thus forming the bottom of 4 identical wells with a volume of $\sim 50 \mu \mathrm{l}$ each. Biomolecular samples for surface functionalization were incubated in still solution. To remove excess sample after each incubation step, the content was diluted by repeated addition of a 2 -fold excess of working buffer and removal of excess liquid until the concentration of the solubilized sample, estimated from the extent of dilution, was below $10 \mathrm{ng} / \mathrm{mL}$ Repeated aspiration and release ensured homogenization of the liquid volume at each dilution step. Care was taken to keep the substrates wet at all times. To avoid unbinding of CXCL12 $\alpha$ from HS films, excess CXCL12 $\alpha$ in solution was not removed and all cell suspensions were supplemented with $5 \mu \mathrm{g} / \mathrm{mL}$ CXCL12 $\alpha$ prior to cell plating.

Prior to plating, cells were re-suspended at a concentration of $10^{6}$ cells $/ \mathrm{mL}$ in RPMI medium without serum. For life cell nuclear labelling, Hoechst 33342 (Sigma Aldrich) at a concentration of $100 \mathrm{ng} / \mathrm{mL}$ was added to the cell suspension. To test for the specificity of the cellular recognition of CXCL12 $\alpha$ through the receptor CXCR4, Tlymphocytes were treated with the human monoclonal anti-CXCR4 antibody 12G5 (R\&D Systems), which inhibits interaction with CXCL12 $\alpha$ [13], at a concentration of $1 \mu \mathrm{g} / \mathrm{mL}$ for $1 \mathrm{~h}$. For assays involving ICAM-1 displaying surfaces, $1 \mathrm{~mm} \mathrm{MgCl} 2$ and $1 \mathrm{~mm}$ EGTA were added to the cell suspension $10 \mathrm{~min}$ before plating; this treatment enhances the affinity of the ICAM-1 cell-surface receptor LFA-1 and thus promotes adhesion to ICAM-1 displaying surfaces [34].

Cells were plated at a density of $2.5 \times 10^{5}$ cells per $\mathrm{cm}^{2}$ on the functionalized glass cover slips. After incubation for $1 \mathrm{~h}$, non-adhesive (and weakly adhesive) cells were removed by gentle rinsing with a pipette in phosphate-buffered saline (PBS; Sigma Aldrich) at pH 7.4. Differential interference contrast (DIC) micrographs and epi-fluorescence micrographs of the nuclear labelling were taken shortly before and after the rinsing step, using an inverted microscope (IX81; Olympus, Hamburg, Germany) equipped with a $60 \times$ oil immersion objective (PlanAPoN60XO; Olympus). At least 20 locations were imaged on each sample, covering a surface area of at least $2 \mathrm{~mm}^{2}$, and used for further quantitative analysis. The same locations were imaged before and after the rinsing step. Volocity visualization software (PerkinElmer, Wlatham, MA, USA) was used for analysis of fluorescence micrographs, to detect the cells and quantify cell surface densities. From a comparison with manual cell counts on selected samples, we estimate the error in the automated determination of cell surface densities to be below 5\%. All assays were repeated at least 4 times with independent cell cultures. The percentage of adherent cells is presented as mean values \pm standard deviation of four independent experiments. To evaluate the statistical significance between the mean values of more than two samples, the ANOVA test with Bonferroni correction was applied, and a $p$-value for $\alpha=0.05$ was extracted for each sample combination.

\section{Results}

\subsection{Design of well-defined biomimetic surfaces}

The design of our biomimetic glycosaminoglycan-presenting surfaces is schematically shown in Fig. 1. Two different approaches were used to immobilize GAGs. Both have in common that they use biotinylated GAGs (here HS) and a monolayer of streptavidin (SAv) on a passivating background that prevents non-specific binding. The passivating background was either a gold-supported oligo(ethylene glycol) (OEG) monolayer exposing biotin at the end of a fraction of the OEG molecules (Fig. 1A) or a silica-supported lipid bilayer (SLB) exposing biotin at the head of a fraction of the lipids (Fig. 1B).

The design rules were chosen such that ensuing molecular interactions give rise to self-assembled yet stable model surfaces that are well-defined and tuneable with regard to the density, the 


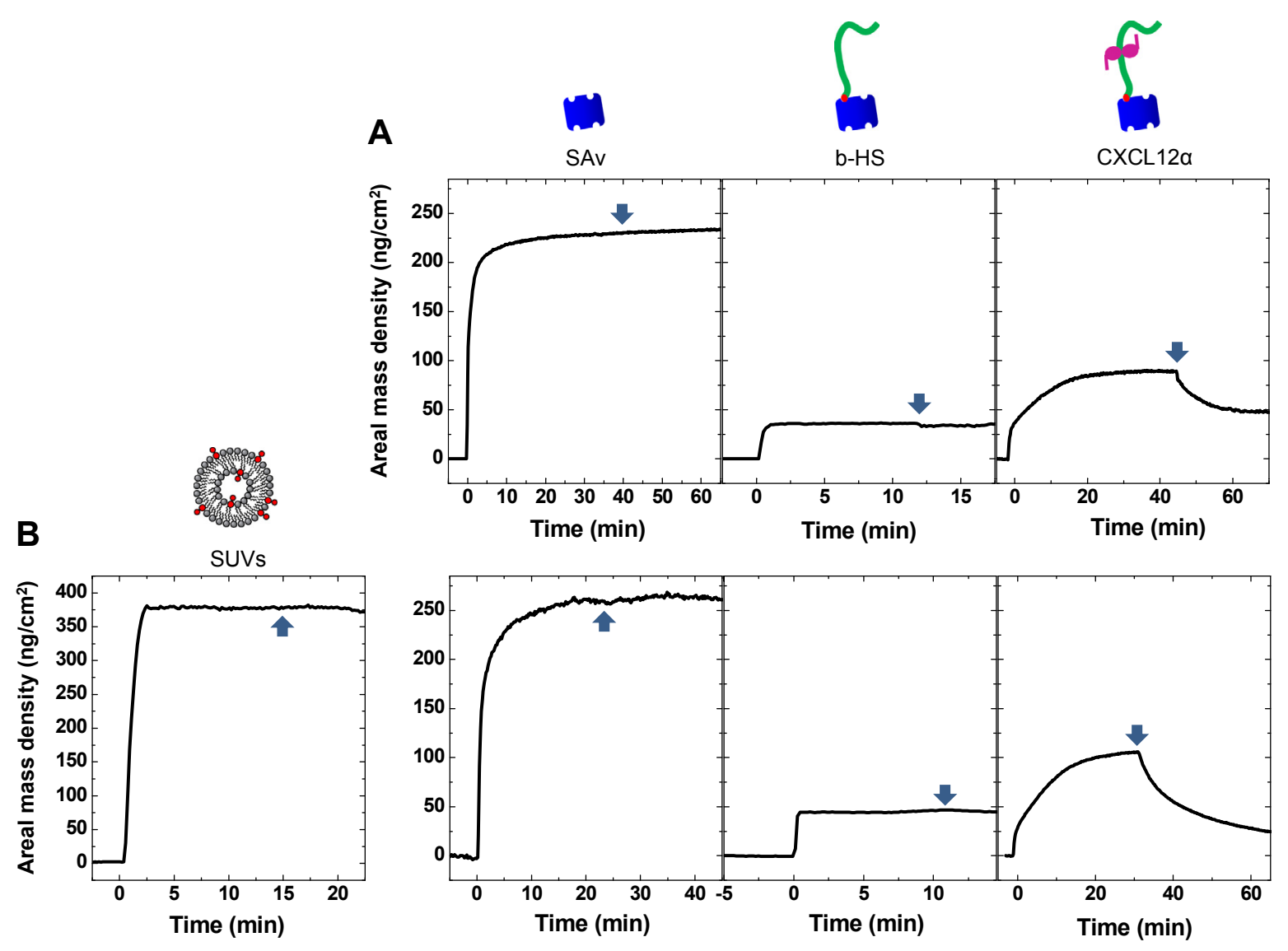

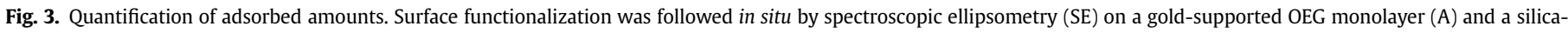

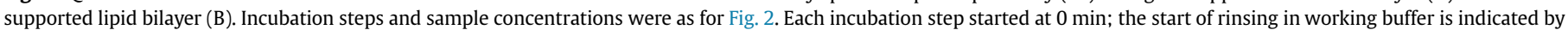
an arrow.

orientation and the lateral mobility of the displayed molecules. In particular, we expect SAv to be immobilized such that two of its four biotin-binding sites are facing the surface for immobilization while the other two binding sites are facing the solution to accommodate target molecules. In this regard, SAv performed better than neutravidin (which is perhaps more frequently used in molecular labelling applications), because neutravidin has an enhanced tendency to aggregate [35] and hence does not form well-defined monolayers. Biotin conjugation of the target molecule at a specific site affords immobilization at a well-defined and desired orientation on SAv monolayers. In the case of HS, for example, site-specific biotinylation at the reducing end ensures binding with an orientation that mimics the attachment to HSdisplaying core proteins in the cell membrane [36]. Moreover, because binding is stoichiometric and very stable, more than one type of biotinylated molecules can be sequentially immobilized on SAv monolayers. Because the bond forms rapidly (i.e. binding is mass-transfer limited at sufficiently low surface densities), surface densities of target molecules can be quantitatively tuned by varying their solution concentrations and incubation times. The main difference between the two passivating backgrounds is the lateral mobility of the SAv molecules. On SLBs, SAv and any target molecule anchored to it can rotate and diffuse laterally (as illustrated by the black arrows in Fig. 1B), provided that the SAv surface density is low enough to prevent two-dimensional protein crystallization $[37,38]$ (the latter was reported to occur at surface densities above $75 \%$ relative to that of the crystalline phase, i.e. above $200 \mathrm{ng} / \mathrm{cm}^{2}$, on lipid monolayers [39]).
To validate our approach, the step-by-step assembly of our biomimetic surfaces was monitored by QCM-D (Fig. 2). OEG monolayers were prepared ex situ prior to installing the goldcoated sensors in the QCM-D chamber. SLBs were formed in situ by the method of vesicle spreading, through incubation of the silica-coated QCM-D sensor with $50 \mu \mathrm{g} / \mathrm{mL}$ SUVs (Fig. 2B). The final shifts in frequency $(\Delta f=-26 \pm 0.5 \mathrm{~Hz})$ and in dissipation $\left(\Delta D \leq 0.3 \times 10^{-6}\right)$ indicates the formation of a confluent SLB of good quality [40]. The binding kinetics upon successive incubation with $1 \mu \mathrm{g} / \mathrm{mL}$ (to check for sample depletion in the fluidic system, see Methods for details) and $20 \mu \mathrm{g} / \mathrm{mL}$ (to rapidly saturate the surface) $\mathrm{SAv}$ were comparable on both surfaces. At equilibrium, SAv generated frequency shifts of $-23 \pm 1 \mathrm{~Hz}$ on OEG monolayers and $-27 \pm 1 \mathrm{~Hz}$ on SLBs, and on both supports, the shifts in dissipation were below $0.4 \times 10^{-6}$. From the frequency shifts, and assuming a mean density of $1.2 \mathrm{~g} / \mathrm{cm}^{3}$ for the SAv film with trapped solvent [41], a film thickness of approximately $4 \mathrm{~nm}$ can be determined using Sauerbrey's equation, consistent with the molecular dimensions of SAv. Overall, these responses are as expected for the formation of rather dense protein monolayers [16,41], in which each SAv molecule exposes two biotin-binding sites each towards the surface and the bulk solution, respectively.

b-HS, incubated at $50 \mu \mathrm{g} / \mathrm{mL}$, readily bound to the free biotinbinding sites on the SAv monolayers, with frequency shifts at equilibrium of $-31 \pm 1 \mathrm{~Hz}$ on OEG monolayers and $-33 \pm 2 \mathrm{~Hz}$ on SLBs, and with corresponding dissipation shifts of $5.0 \pm 0.5 \times 10^{-6}$ and $5.5 \pm 0.5 \times 10^{-6}$, respectively. These responses indicate the formation of a soft and presumably highly hydrated film. The HS 

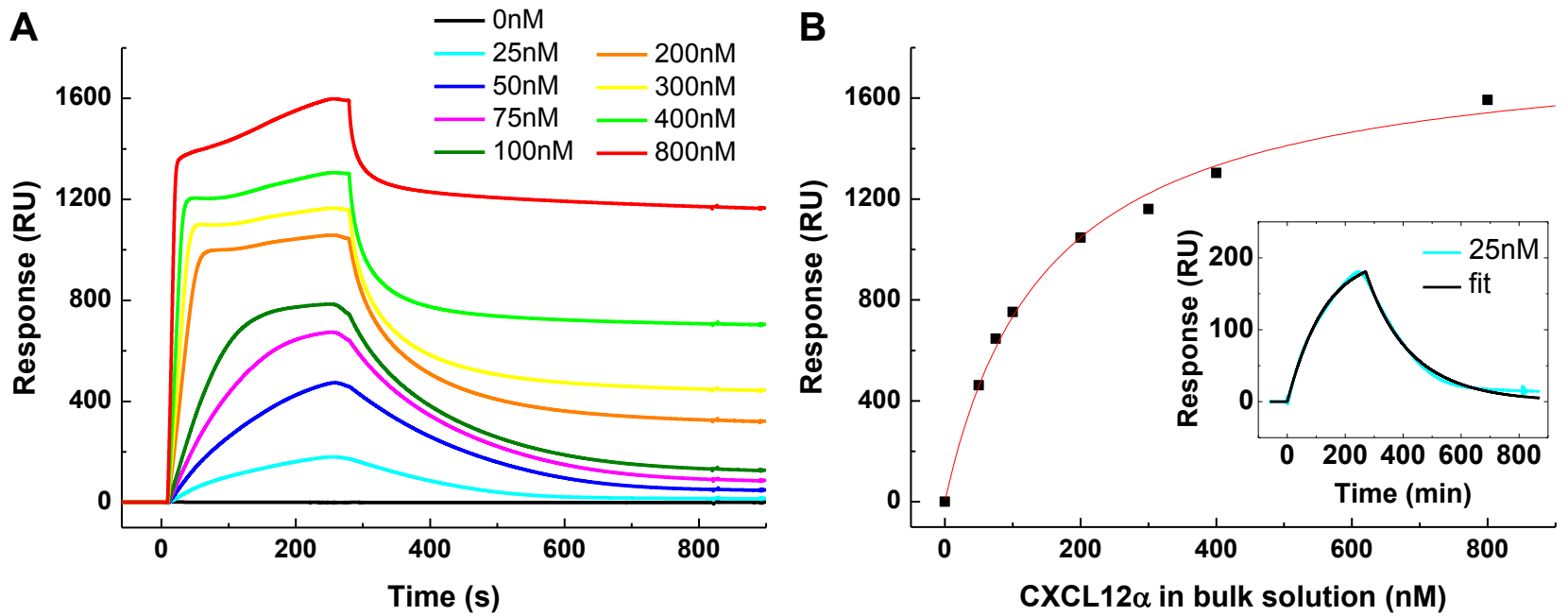

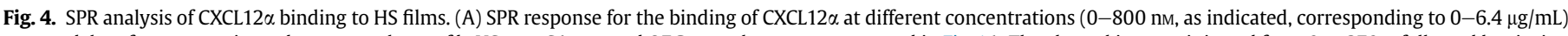

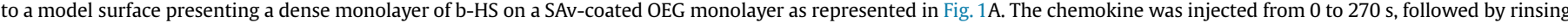

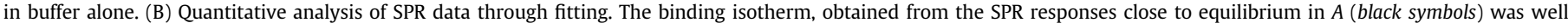

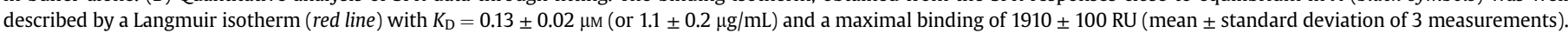

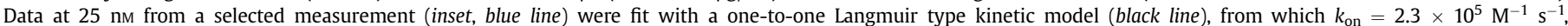

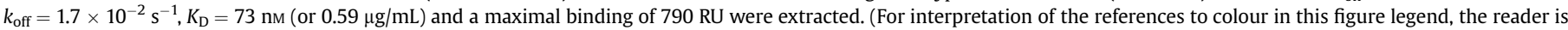
referred to the web version of this article.)

film was completely stable to rinsing in buffer, as expected for immobilization through the strong biotin-SAv bond. We note that the HS surface densities obtained in the present measurements correspond to the maximal attainable coverage. Lower surface densities can be readily obtained by tuning b-HS incubation time and concentration. By fitting the QCM-D responses to a viscoelastic model, the effective thickness of the HS films was estimated to be $11.6 \pm 1.2 \mathrm{~nm}$. In comparison, the average contour length of the employed HS chains is approximately $25 \mathrm{~nm}$. The QCM-D data are hence consistent with the formation of a film of end-grafted HS, in which the individual chains are slightly coiled and/or point in random directions with respect to the surface normal.

The chemokine CXCL12 $\alpha$, incubated at $5 \mu \mathrm{g} / \mathrm{mL}$, generated negative shifts in frequency ( $-9 \pm 1 \mathrm{~Hz}$ on both surfaces), indicating binding. The strong concomitant decrease in dissipation (by $-5 \pm 1 \times 10^{-6}$ on OEG monolayers and $-4.4 \pm 1 \times 10^{-6}$ on SLBs) indicates protein-induced rigidification of the HS film. This remarkable effect merits further investigation, which will be the subject of a forthcoming study. Upon subsequent rinsing in buffer, frequency and dissipation increased slowly but did not return to the level of the virgin HS film, demonstrating that some CXCL12 $\alpha$ was released over experimentally accessible time scales whereas a sizeable fraction remained rather stably bound and displayed by HS.

We performed several additional assays to validate the quality of our surfaces and the specificity of immobilization. Bovine serum albumin (BSA) at $100 \mu \mathrm{g} / \mathrm{mL}$ did not bind to the OEG monolayer (Fig. 2A), confirming that the OEG film indeed effectively blocks against access of proteins to the underlying gold surface. BSA also did not bind to the SLB or to the SAv monolayers (not shown). HS lacking biotin functionality did not bind to any of the SAv monolayers (Fig. 2C and E), confirming that b-HS is exclusively immobilized through the biotin moiety at the GAG's reducing end. CXCL12 $\alpha$ did not bind any of the SAv monolayers in the absence of HS (Fig. 2D and F), confirming that chemokine binding to HS is specific. Moreover, the CXCL12 $\alpha$ fraction that remained in the HS film after rinsing with buffer could be eluted, and the HS film thus fully regenerated, by exposure of the surfaces to $2 \mathrm{~m}$ of either guanidine hydrochloride (GuHCl; Fig. S1) or $\mathrm{NaCl}$ (not shown).

\subsection{Quantitative analysis of surface densities of functional} biomolecules

The surface densities of biomolecules during the step-by-step assembly of the biomimetic surfaces were quantified by spectroscopic ellipsometry (SE; Fig. 3). As for the QCM-D measurements, OEG monolayers were formed ex situ, whereas all other immobilization steps were followed in situ. Time-resolved data for OEG monolayers and SLBs are displayed in Fig. 3A and B, respectively, and Table 1 provides quantities extracted at equilibrium. The lipid surface density at equilibrium $\left(380 \mathrm{ng} / \mathrm{cm}^{2}\right)$ is consistent with expectations for an SLB. At the maximal attained SAv coverage on SLBs, the average surface area available per SAv molecule was $\sim 37 \mathrm{~nm}^{2}$. In comparison, the projected surface area of an appropriately oriented $\mathrm{SAv}$ molecule was estimated to $4.3 \mathrm{~nm} \times 5.4 \mathrm{~nm} \approx 23 \mathrm{~nm}^{2}$ [41], confirming that SAv formed a dense monolayer. Consistent with expectations from the QCM-D frequency responses (Fig. 1A-B), OEG monolayers could accommodate a similar yet slightly (18\%) lower density of SAv, corresponding to an average surface area per molecule of $\sim 45 \mathrm{~nm}^{2}$. The increased binding on SLBs could be due to the lateral mobility of SAv on fluid SLBs, allowing reorganization into a more densely packed monolayer. Taken together, with two biotin-binding sites available per SAv molecule, the average surface area per biotinbinding site (i.e. anchor point for b-HS) on saturated SAv monolayers would be $21 \pm 2 \mathrm{~nm}^{2}$, corresponding to an average spacing of $4.5 \mathrm{~nm}$ (assuming packing in a square lattice).

The binding curves for b-HS in Fig. 3 reveal a constant binding rate up to approximately $80 \%$ of maximal coverage, and a rapid saturation thereafter. This indicates that HS binding is masstransfer limited at low surface densities [42], and that kinetic limitations due to crowding of HS chains on the surface do only weakly affect HS binding even at high surface densities. This would suggest that all solution-facing biotin-binding sites (i.e. two per SAv molecule) can be occupied with b-HS. According to Table 1, and considering a SAv molecular mass of $60 \mathrm{kDa}$ [41], the amount of HS bound on average per biotin-binding site is $5.2 \pm 0.6 \mathrm{kDa}$. This value is inferior to the average HS molecular mass employed (12 $\mathrm{kDa})$. 
Table 1

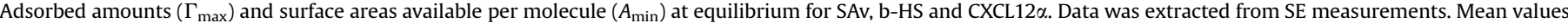

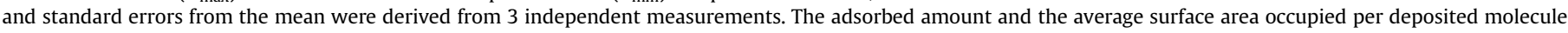
were estimated to quantify the number of SAv or HS molecules grafted per unit surface area, and the stoichiometry of chemokine binding.

\begin{tabular}{|c|c|c|c|c|c|c|}
\hline \multirow[t]{3}{*}{ Immobilization platform } & \multicolumn{2}{|l|}{ SAv } & \multicolumn{2}{|l|}{ b-HS } & \multicolumn{2}{|l|}{ CXCL12 $\alpha$} \\
\hline & $\Gamma_{\max }$ & $A_{\min }$ & $\Gamma_{\max }$ & $A_{\min }$ & $\Gamma_{\max }$ & $A_{\min }$ \\
\hline & $\overline{\left(\mathrm{ng} / \mathrm{cm}^{2}\right)}$ & $\left(\mathrm{nm}^{2}\right)$ & $\left(\mathrm{ng} / \mathrm{cm}^{2}\right)$ & $\left(\mathrm{nm}^{2}\right)$ & $\overline{\left(\mathrm{ng} / \mathrm{cm}^{2}\right)}$ & $\left(\mathrm{nm}^{2}\right)$ \\
\hline OEG monolayer on gold & $235 \pm 6$ & $42.4 \pm 1.1$ & $35.5 \pm 2.2$ & $56.3 \pm 3.5^{\mathrm{a}}$ & $78 \pm 7$ & $17.4 \pm 1.6$ \\
\hline SLB on silica & $273 \pm 8$ & $36.5 \pm 1.5$ & $46.8 \pm 1.5$ & $42.6 \pm 1.4^{\mathrm{a}}$ & $120 \pm 20$ & $11.5 \pm 1.9$ \\
\hline
\end{tabular}

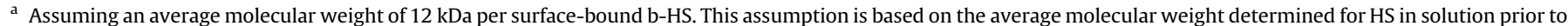

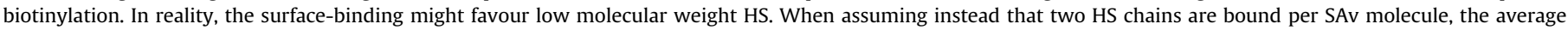

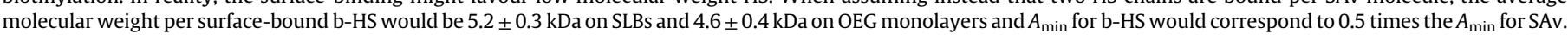

The discrepancy is likely a consequence of the large size distribution of HS in solution, i.e. capture on SAv has selected the shortest chains in the initial HS sample. The binding of smaller molecules tends to be favoured, because of their faster diffusion and hence mass transfer to the surface [42] and because they may also penetrate an existing HS film more easily. Assuming an average molecular mass of an HS disaccharide of 500-550 Da [43], we can estimate that $10 \pm 2$ disaccharides are bound on average per biotinbinding site. With a length of $1.0 \mathrm{~nm}$ per disaccharide, the average chain contour length would then be $10 \mathrm{~nm}$. The final b-HS surface density on OEG monolayers was slightly lower than on SLBs. This is most likely a consequence of the reduced SAv density on OEG monolayers. Indeed, within the experimental uncertainties, the mass ratio of b-HS to SAv was constant on both surfaces.

The binding curve for CXCL12 $\alpha$ reproduced many features already observed by QCM-D (Fig. $2 \mathrm{~A}-\mathrm{B}$ ), such as rapid binding and equilibration and partial release of proteins upon rinsing in buffer. Thanks to the quantification of surface densities afforded by SE (Table 1), and with a CXCL12 $\alpha$ molecular mass of $8.1 \mathrm{kDa}$, it is possible to estimate that each CXCL12 $\alpha$ molecule has approximately 3.5 kDa HS, corresponding to roughly 7 disaccharides, available on average at equilibrium.

\subsection{Application of the biomimetic GAG-presenting surfaces to molecular interaction analysis}

Surface plasmon resonance (SPR) was used to analyse thermodynamic and kinetic parameters of the interaction of CXCL12 $\alpha$ with HS films (Fig. 4). SPR studies of CXCL12 $\alpha$ binding to HS have previously been reported [9] using a film of carboxymethylated dextran (i.e. Biacore CM4 sensor chips) with covalently immobilized SAv as an immobilization platform. The dextran film is approximately $100 \mathrm{~nm}$ thick [44] and hence represents a threedimensional environment into which b-HS was bound (at unknown volume densities). The model surfaces in our study are distinct, in that all HS molecules are presented at the same orientation in the form of a monolayer with controlled surface density. As for QCM-D (Fig. 2A) and SE (Fig. 3A) measurements, the goldcoated SPR sensor chip was first functionalized ex situ with an OEG monolayer, and subsequent functionalization was monitored in situ. At saturation, $2550 \pm 25$ and $330 \pm 20$ response units (RU) were reached for SAv and b-HS, respectively. According to Table 1, these values correspond to surface densities of approximately 235 and $35 \mathrm{ng} / \mathrm{cm}^{2}$, respectively.

The sensorgram in Fig. 4A shows a response in CXCL12 $\alpha$ binding that is dose dependent in the initial binding rates and the binding equilibrium, as expected. For low protein concentrations $(\leq 100 \mathrm{~nm})$, the binding curves were conventional and the protein could be close-to-completely removed upon rinsing in buffer. The unbinding curves at the lowest employed concentration ( $25 \mathrm{nM}$ ) were well-fitted by a one-to-one Langmuir type binding model
(Fig. 4B, inset), revealing an association rate constant $k_{\text {on }}=2.3 \times 10^{5} \mathrm{M}^{-1} \mathrm{~s}^{-1}$, a dissociation rate constant $k_{\text {off }}=1.7 \times 10^{-2} \mathrm{~s}^{-1}$, and hence a dissociation constant $K_{\mathrm{D}}=k_{\mathrm{off}} /$ $k_{\text {on }}=73 \mathrm{~nm}$ (or $0.59 \mu \mathrm{g} / \mathrm{mL}$ ), and a maximal response of $790 \mathrm{RU}$. The results of the fit varied somewhat depending on how much of the unbinding curve was included in the fit, and from these variations we estimate the $K_{\mathrm{D}}$ to be accurate to within a few $10 \%$. The simple one-to-one binding model increasingly failed to reproduce the experimental data with increasing protein concentration. Above $100 \mathrm{~nm}$, a peculiar multi-phase binding response appeared: a first fast binding was followed by a quasi-plateau and a phase of slower binding before equilibrium was reached. Moreover, a substantial fraction of the chemokine remained bound upon rinsing in buffer at these protein concentrations. The multi-phase binding response and the limited release appear to be correlated, suggesting that CXCL12 $\alpha$ can bind to HS in at least two distinct ways.

The complex binding pattern precluded a further quantitative analysis of the kinetic SPR data. However, a binding isotherm was constructed from the SPR responses close to equilibrium (Fig. 4B). The data could be fitted with a Langmuir isotherm with $K_{\mathrm{D}}=0.13 \pm 0.02 \mu \mathrm{m}($ or $1.1 \pm 0.2 \mu \mathrm{g} / \mathrm{mL})$ and a maximal response of $1910 \pm 100 \mathrm{RU}$, both significantly larger though of the same order of magnitude as the values obtained above for a solution concentration of $25 \mathrm{~nm}$. Considering the complex binding pattern observed by SPR, and the heterogeneous sulphation of HS, it is rather surprising that the binding isotherm is so well described by the simple Langmuir binding model. The agreement might well be coincidental, and we therefore suggest that the $K_{\mathrm{D}}=0.13 \mu \mathrm{m}$ obtained through the binding isotherm should be considered an effective value rather than representing the true binding affinity of a one-toone binding interaction. Notably, our effective $K_{\mathrm{D}}$ value is comparable to the value of $0.20 \mu \mathrm{m}$, previously obtained by SPR with a film of HS-functionalized carboxymethylated dextran using an HS preparation of comparable sulphation and molecular weight [9]. The $K_{\mathrm{D}}=73 \mathrm{nM}$ obtained through analysis of the kinetic data at $25 \mathrm{nM}$, on the other hand, may be a true binding constant, representing the highest-affinity binding sites in the HS film. Since CXCL12 $\alpha$ tends to bind to highly sulphated regions [47], one would expect this number to be comparable to the affinity of CXCL12 $\alpha$ for the highly sulphated GAG heparin. Indeed, a similar value of $93 \mathrm{nM}$ has been reported for heparin [9].

We note that the SPR responses at equilibrium upon CXCL12 $\alpha$ binding (Fig. 4A) were several fold larger than what was previously reported on CM4 sensor chips [9]. Most likely, this is due in part to a higher HS density selected in our assays and in another part to an enhanced sensitivity of our assay (i.e. because the SPR sensitivity decays exponentially with the distance from the gold surface, and the interactions in our assay are confined to within about $20 \mathrm{~nm}$ whereas the CM4 chip samples approximately $100 \mathrm{~nm}$ ). By comparing the SE data for CXCL12 $\alpha$ binding (at $5 \mu \mathrm{g} / \mathrm{mL}$ or $620 \mathrm{nM}$ solution concentration; Table 1) with the Langmuir isotherm 
A
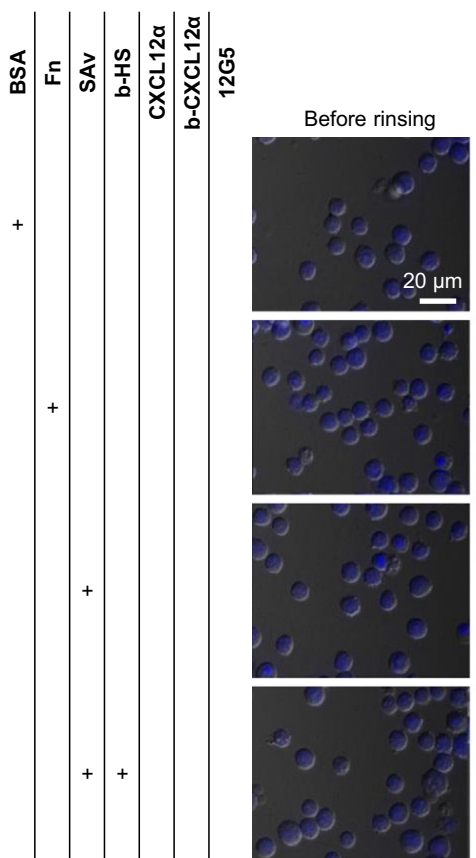

$+\quad \mid \begin{gathered}\vec{\xi} \\ \frac{\partial}{2} \\ \text { م) }\end{gathered}$

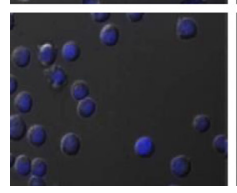

After rinsing
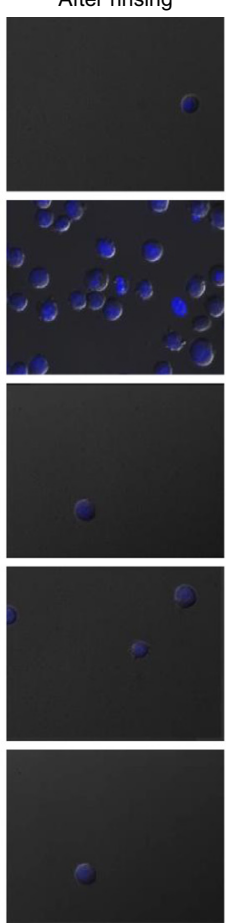

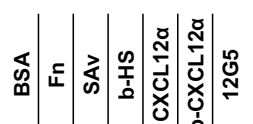

Before rinsing

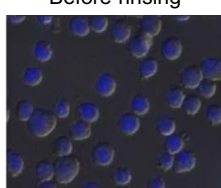

After rinsing
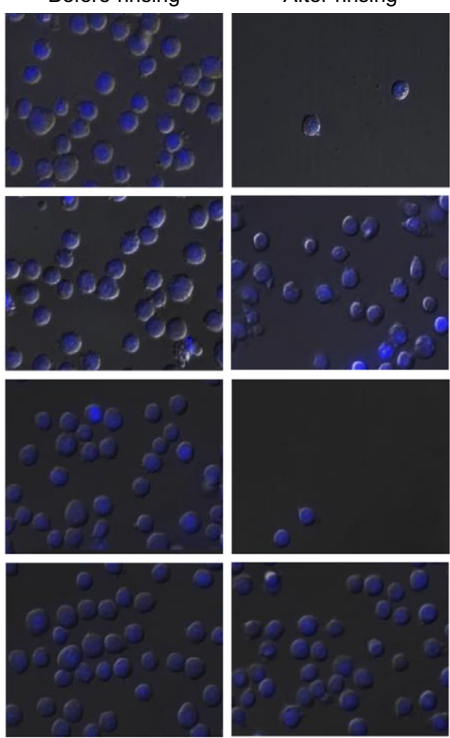

$+$
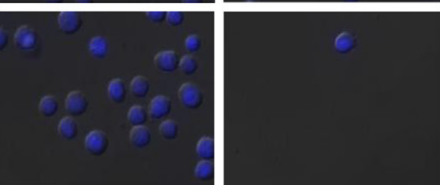

B

$\mathrm{p}=5^{*} 10^{-9}$

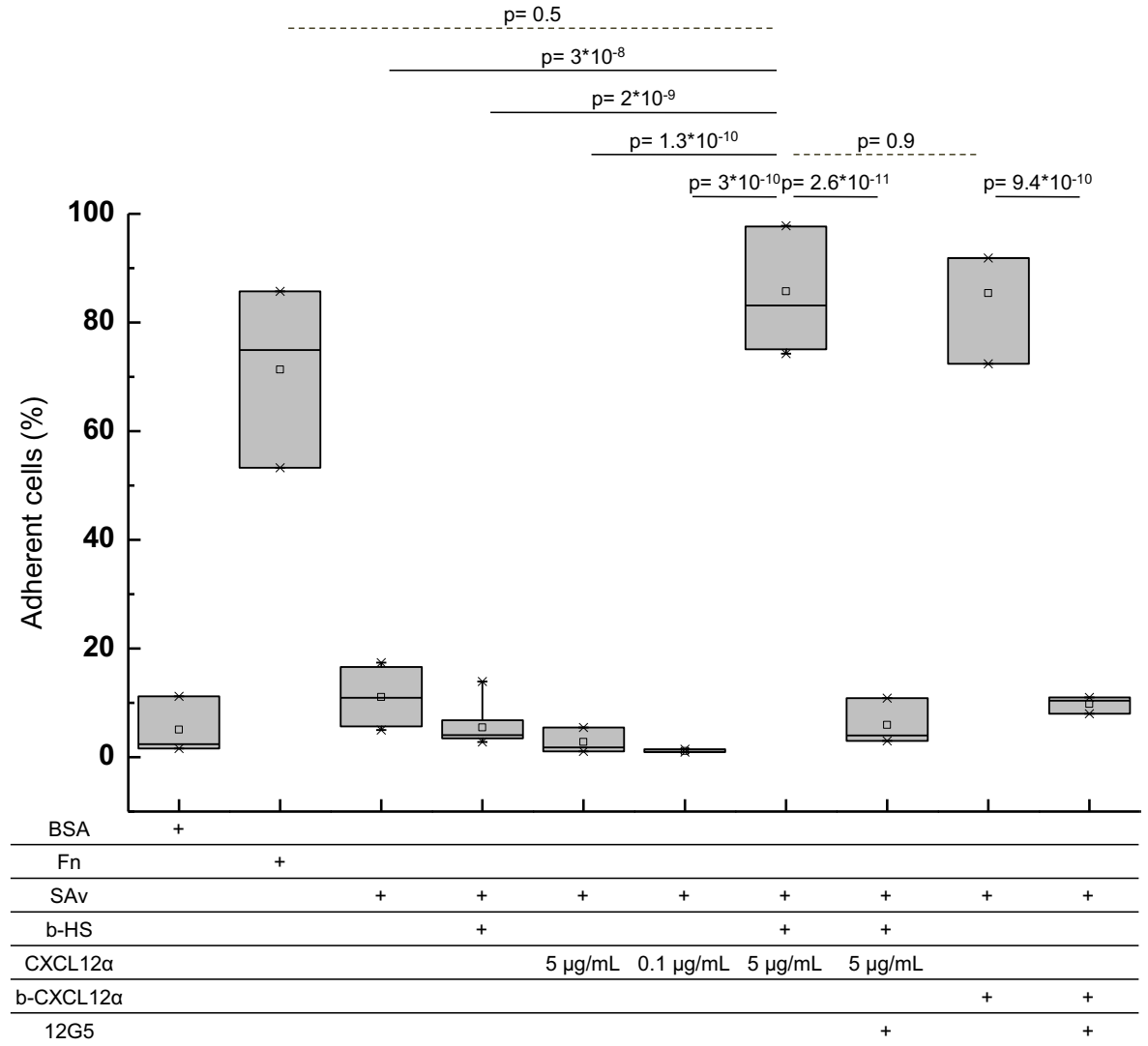

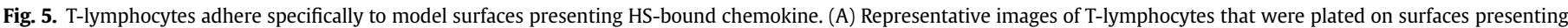

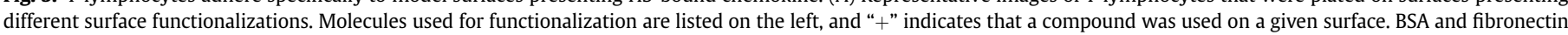

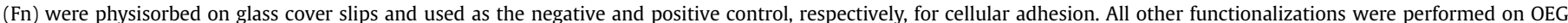

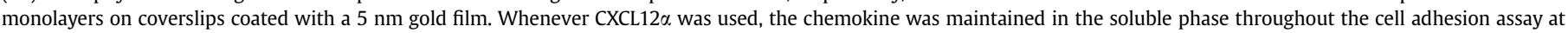


derived from SPR (Fig. 4B), we can estimate that the maximal SPR response of $1910 \mathrm{RU}$ corresponds to roughly 6 disaccharides available on average per CXCL12 $\alpha$. CXCL12 $\alpha$ is known to dimerize upon binding to GAGs, with the GAG binding site being located at the interface between the constituent monomers [45-47], i.e. 12 disaccharides would be effectively available per CXCL12 $\alpha$ dimer binding site. In comparison, structural models and binding data have suggested that a CXCL12 $\alpha$ dimer occupies approximately 6 disaccharides [47,50]. Thus, if all CXCL12 $\alpha$ are bound directly to HS and if CXCL12 $\alpha$ binds exclusively to the highly sulphated regions, then this would mean that about one half of the HS is highly sulphated. The degree of sulfation in HS is diverse and depends on the source, but the above calculation is clearly in the range of what is possible.

\subsection{Application of the biomimetic GAG-presenting surfaces to cellular interaction studies}

Increasing complexity, the biomimetic GAG-presenting surfaces were used to trigger specific cellular responses. As a model system, we chose CXCL12 $\alpha$-loaded HS-presenting surfaces and Jurkat cells as a CXCL12 $\alpha$ sensitive T-lymphocytes cell line [13]. In a first step, the adhesion of Jurkat cells to surfaces with different functionalizations was assessed by quantifying the fraction of cells that resisted gentle rinsing with a pipette after $1 \mathrm{~h}$ of exposure to the surface (Fig. 5). Less than $20 \%$ of cells adhered stably to glass cover slips with physisorbed BSA whereas more than 60\% of cells remained attached on glass cover slips with physisorbed fibronectin (Fn). These surfaces served as negative and positive controls, respectively.

All other functionalizations were performed on OEG monolayers on coverslips coated with a $5 \mathrm{~nm}$ (i.e. semi-transparent) gold film, following the previously established protocol (Fig. 1A). Surfaces displaying a virgin SAv monolayer or a SAv monolayer with HS film showed a level of cellular adhesion that was comparable to the negative control (Fig. 5). This demonstrates that our surfaces are resistant to non-specific cellular adhesion, as desired. The presence of CXCL12 $\alpha$ at $100 \mathrm{ng} / \mathrm{ml}$ in the bulk solution did not enhance cell adhesion to a virgin SAv monolayer. At this chemokine concentration, close to CXCL12 $\alpha$ plasma concentration during inflammation [48], T-lymphocytes are known to become activated [49]. Even with CXCL12 $\alpha$ at $5 \mu \mathrm{g} / \mathrm{mL}$ in the bulk solution, T-lymphocyte adhesion remained at baseline level on virgin SAv monolayers. We conclude that stimulation through CXCL12 $\alpha$ in the solution does not promote significant (non-specific) cell adhesion.

In contrast, when CXCL12 $\alpha$ was presented by the surface through HS (Fig. 5), cellular adhesion increased significantly, to levels that were comparable or even superior to Fn-displaying surfaces. Considering that CXCL12 $\alpha$ is not known as a cell adhesion ligand and that $\mathrm{CXCL} 12 \alpha$ binds reversibly to the HS-coated surface, this finding is surprising. When CXCL12 $\alpha$-binding to its cell-surface receptor CXCR4 was blocked with the anti-CXCR4 antibody 12G5 [13], cell adhesion returned to baseline levels, demonstrating that CXCL12 $\alpha$-mediated adhesion of Jurkat cells to HS-presenting surfaces is specific and mediated by CXCR4.

In a complementary assay, we tested if the presence of HS was required for CXCL12 $\alpha$-mediated cellular adhesion (Fig. 5). To this end, CXCL12 $\alpha$ was immobilized directly on the SAv monolayer using a biotinylated protein construct (Fig. S2). Site-specific conjugation with a biotin at position 68 , the C-terminal residue that is not expected to interfere with CXCL12 $\alpha$ binding to HS (see Fig. S2A) or to the cell surface receptor CXCR4 [50], ensured close-to-stoichiometric binding of $\mathrm{CXCL} 12 \alpha$ to SAv (i.e. two CXCL12 $\alpha$ molecules per SAv, Fig. S2B) at appropriate orientation. There was no significant difference in the cellular adhesion between the two methods of CXCL12 $\alpha$ presentation (Fig. 5B; $p$ value $=0.9$ ). Therefore, under the employed conditions (including CXCL12 $\alpha$ surface densities of similar magnitude, see Fig. 3A and Fig. S2B), the presentation of the chemokine through HS does apparently not alter the cellular response in terms of adhesion to CXCL12 $\alpha$ as compared to chemokine presentation in the absence of HS.

\subsection{Cell adhesion on surfaces presenting GAGs together with cell adhesion ligands - towards more complex cellular interaction studies}

With its modular design, our surface functionalization platform can readily accommodate multiple biotinylated compounds, generating multifunctional surfaces (Fig. 1A). To demonstrate this, surfaces were created on which the intercellular adhesion molecule 1 (ICAM-1) was immobilized, either alone or in combination with HS. ICAM- 1 is known to be presented by the endothelial cell surface and to bind to the leucocyte function-associated antigen-1 (LFA-1; also called integrin $\alpha_{L} \beta_{2}$ ). This interaction is responsible for the attachment of T-lymphocytes to the vascular endothelium, a precursor step towards migration through the endothelial barrier [51]. Here, we tested how the co-presentation of HS-bound CXCL12 $\alpha$ and ICAM- 1 affects cell adhesion.

The assembly of the new model surface was first characterized by QCM-D. A fusion protein made from two ICAM-1 extracellular domains and an immunoglobulin Fc domain (FC-ICAM-1) could be immobilized stably and with desired orientation through an adaptor protein (b-ZZ) that contained two Z-fragments of Protein A (for binding to the Fc domain) and a site-specifically conjugated biotin (for binding to SAv) (Fig. S3). Fig. 6A confirms that b-HS and $b-Z Z$ can be sequentially immobilized on the same SAv monolayer, and that the resulting surface can be used to codisplay CXCL12 $\alpha$ (specifically through b-HS, see also Fig. S3A) and Fc-ICAM-1 (specifically through b-ZZ, see also Fig. S3B). For the cell adhesion assays, surfaces presenting HS-bound CXCL12 $\alpha$ together with ICAM-1 were compared with surfaces presenting either HS-bound CXCL12 $\alpha$ or ICAM-1 alone. Surfaces displaying b-ZZ, either alone or with HS, were used as negative controls. Throughout the assay, surface densities of the incubated components were maintained constant. The surface coverage of b-HS and b-ZZ was controlled by varying the samples' solution concentrations and incubation times and exploiting the fact that binding to SAv is mass transport limited at sufficiently low b-HS and b-ZZ surface densities (Fig. S4). The surface density of b-HS was fixed to $35 \pm 5 \%$ of the maximal surface density (Fig. S4A), corresponding to an average distance of about $13 \mathrm{~nm}$ between neighbouring HS chains. Onto this low-density HS film, CXCL12 $\alpha$ bound with an equilibrium surface density of $35 \pm 4 \mathrm{ng} / \mathrm{cm}^{2}$ (Fig. S4B). b-ZZ was immobilized at $7 \mathrm{ng} / \mathrm{cm}^{2}$ (Fig. S4C). This surface density, corresponding to an average distance of $28 \mathrm{~nm}$

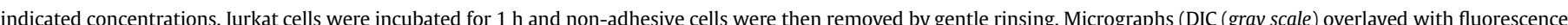

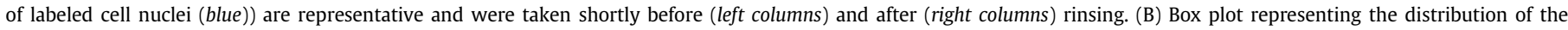

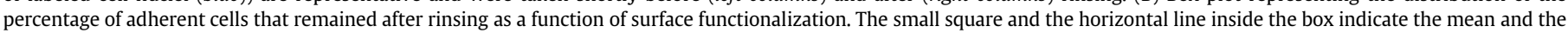

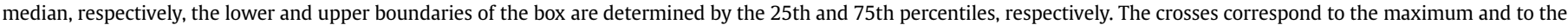

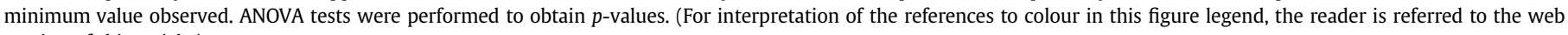
version of this article.) 

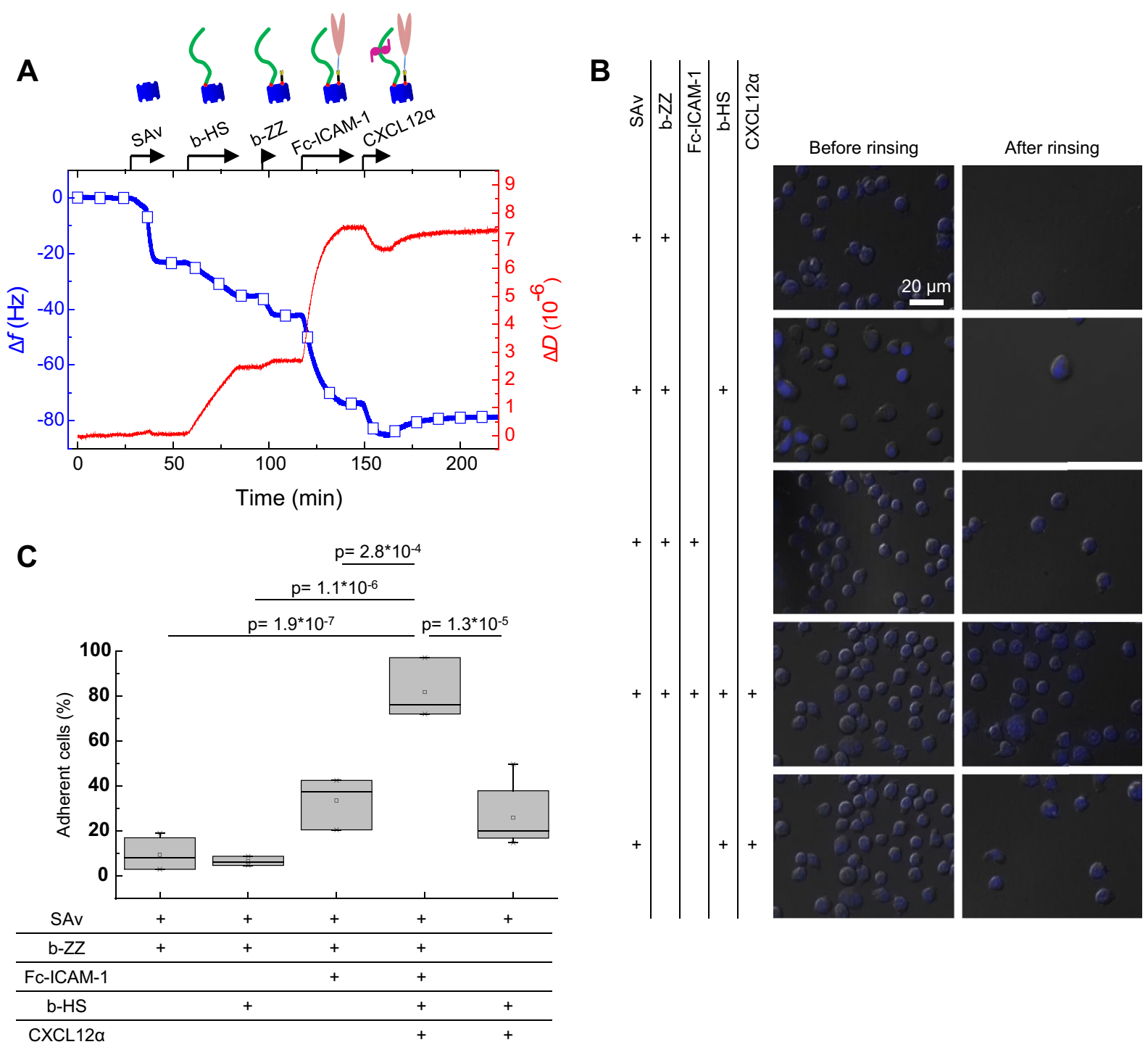

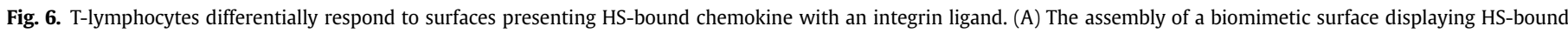

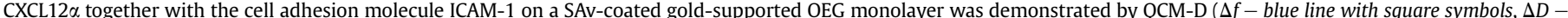

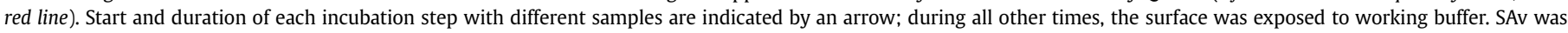

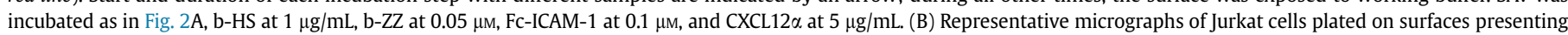

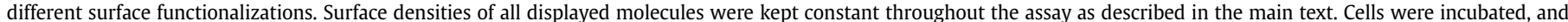

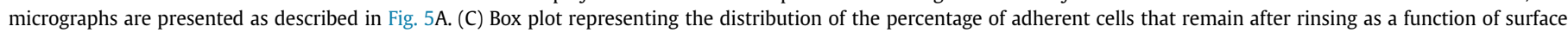
functionalization (analysis analogous to Fig. 5B). (For interpretation of the references to colour in this figure legend, the reader is referred to the web version of this article.)

between neighbouring anchor points for Fc-ICAM-1, was chosen to have an ICAM-1 surface density not too high to be able to appreciate the effect of the co-presentation of the integrin ligand with the chemokine presented through HS.

Considering the molecular dimensions of the Fc-ICAM-1 construct - the five immunoglobulin (Ig)-like domains per each of the two ICAM-1 domains [52] and the four Ig domains of the Fc part [53] are expected to form an assembly of two bent rods aligned at the Fc part, where each rod is about $26 \mathrm{~nm}$ long and $2 \mathrm{~nm}$ in diameter - it is unlikely that steric constraints will limit the binding of Fc-ICAM-1 to b-ZZ, and the average distance between ICAM-1 dimers is therefore estimated to be also $28 \mathrm{~nm}$. Moreover, with the chosen surface densities, surface crowding should not limit immobilization of any of the molecular species. In contrast to the previous assay (Fig. 5), Jurkat cells were treated with $1 \mathrm{~mm} \mathrm{MgCl}_{2}$ and $1 \mathrm{~mm}$ EGTA to induce high-affinity binding of LFA- 1 to ICAM-1 and thus to enhance T-lymphocyte adhesion $[54,55]$.
Results are presented in Fig. 6B-C. Only 10\% of the cells adhered to the negative control surfaces, comparable to the BSA control previously used (Fig. 5B), confirming low non-specific binding. A larger fraction of cells, about $34 \%$ and $26 \%$, adhered to surfaces displaying either Fc-ICAM-1 or HS-bound CXCL12 $\alpha$ alone, respectively. Interestingly, the adhesion increased drastically, to about $80 \%$, when ICAM- 1 and HS-bound CXCL12 $\alpha$ were presented together. This assay thus demonstrates that the co-presentation of an integrin ligand and a GAG-bound chemokine elicits a cellular response that is distinct from the response to each individual cue alone.

\section{Discussion}

We have developed a bottom-up biosynthetic approach to reconstitute GAGs and other cell membrane and extracellular matrix molecules (lipids and proteins) into well-defined model surfaces and demonstrated the application of these tailor-made 
biomimetic environments for quantitative molecular and cellular studies.

The presented surface functionalization strategy is versatile. SAv monolayers serve as a molecular breadboard for the selective coupling of various biomolecules. SAv acts as a host for biotin which is site-specifically conjugated, either directly to the desired biomolecule (e.g. Fig. 2 and Fig. S2) or to an adaptor molecule that binds other tags. The latter was demonstrated here with $\mathrm{b}-\mathrm{ZZ}$ and an Fc chimera (Fig. S3) and other highly specific yet stable interactions can also be exploited (e.g. multivalent NTA constructs and histidine tags [56]). Moreover, the surface density - and hence also the average molecular spacing - can be controlled (Fig. 3) and tuned (Fig. S4). Although not explicitly demonstrated in this study, the design principle also enables comparative studies on surfaces displaying immobile (Fig. 1A) vs. laterally mobile (Fig. 1B) molecules, e.g. to assess the importance of ligand clustering in cellular interactions. Taken together, surface functionalization combined with the design of molecular building blocks through synthetic conjugation chemistry or biochemistry thus provide a toolbox of interactions for the assembly of multifunctional surfaces in a molecular-lego-type fashion.

The employed surface design, validated by QCM-D and SE characterization, confers control on molecular orientation such that the appropriate molecular face is exposed to the solution phase. This ensures that the vast majority of immobilized molecules remains active, in contrast to conventional immobilization approaches such as physisorption (e.g. in enzyme-linked immunosorbent assays (ELISA)) or covalent coupling through random sites (e.g. carboxylic acids or primary amines via EDC NHS chemistry), where surface-induced denaturation and/or spatial constraints can drastically limit the activity of immobilized molecules [57,58]. Biospecific interactions are also more rapid than practically relevant covalent chemistries (including the socalled 'click' chemistries), thus enabling rapid assembly of the biomimetic surfaces. The interactions between biotin and SAv, or between $\mathrm{Fc}$ and $\mathrm{Z}$ domains, are strong enough for the surfaces to remain stable over many hours. Where required, such noncovalent yet rapid and highly specific interactions could be exploited for initial coupling to guide the subsequent formation of covalent bonds at desired sites with enhanced rates [59], thereby enhancing stability and further broadening the application range.

Several proof-of-concept measurements illustrated the use of the biomimetic surfaces for mechanistic studies. On the molecular scale, we find that CXCL12 $\alpha$ may bind to HS in several distinct ways (Fig. 4). CXCL12 $\alpha$ is known to dimerize upon binding to GAGs [45-47], and higher-order oligomers of this chemokine have also been reported [60]. Moreover, HS are heterogeneously sulphated and CXCL12 $\alpha$ is known to bind preferentially to the highly sulphated domains [47]. All these factors might contribute to the complex binding behaviour, in a way that remains to be elucidated. We also find that CXCL12 $\alpha$ rigidifies HS films (Fig. 2A-B), indicating that the interaction of this chemokine affects the supramolecular arrangement of HS chains. In future studies, the model surfaces should be versatile towards elucidating the molecular mechanism behind GAG matrix remodelling by CXCL12 $\alpha$ and its functional consequences. More generally, it becomes possible to study directly on the supramolecular scale how extracellular proteins bind to GAG matrices and remodel them, or how the presentation of GAGs affects protein retention and dynamics (e.g. towards the formation of chemokine gradients $[24,61])$.

At the cellular level we demonstrate that the specific interaction between HS-bound CXCL12 $\alpha$ and the receptor CXCR4 promotes Tlymphocyte adhesion (Fig. 5). Given that the interaction between
HS and CXCL12 $\alpha$ is reversible and considering that no other bona fide cell adhesion receptor is involved, this finding is remarkable. It suggests that CXCL12 $\alpha$ can interact simultaneously and in trans with HS and CXCR4 and that this interaction is strong enough to confer adhesion. The CXCL12 $\alpha$-mediated bridging of CXCR4 and HS is consistent with the observation that in CXCL12 $\alpha$, the binding domains for GAG and CXCR4 are spatially distant and do not interfere functionally [62].

Although the presentation of CXCL12 $\alpha$ through HS and in the form of b-CXCL12 $\alpha$ is distinct - HS displays CXCL12 $\alpha$ in dimeric form [45-47] and reversibly bound whereas b-CXCL12 $\alpha$ is monomeric and irreversibly immobilized - no significant difference in the adhesion of T-lymphocytes was found. This observation might suggest that the specific conformation in which CXCL12 $\alpha$ is presented is not crucial for chemokine recognition by T-lymphocytes. It has been demonstrated that, when presented in solution, both monomeric and dimeric CXCL12 $\alpha$ are recognized by CXCR4. However, the oligomerization state has antagonistic effects on cell signalling and function [63]: low monomer concentrations enhanced chemotaxis while the dimer inhibited chemotaxis $[64,65]$. The assays here developed enable the presentation of CXCL12 $\alpha$ in a matrix-bound form, mimicking in this way the endothelial cell surface, and thus provide means to test how distinct presentations of CXCL12 $\alpha$ in a matrix-bound form affect cellular response. Future studies should investigate if the display of CXCL12 $\alpha$ through an HS matrix, and the potential internalization of the reversibly HS-bound chemokine by the cell, leads to distinct downstream effects that are not detectable in the simple cell adhesion assay used here. It will also be interesting to analyse how the oligomerization state of CXCL12 $\alpha$ affects the cellular response. Moreover, assays with distinct GAG conjugates would enable to study how the HS sulphation pattern or the GAG type affect chemokine-mediated cellular responses.

While integrins and L-selectin are recognized as the major adhesion receptors expressed on the surface of $T$ cells [51], it is known that signals from homeostatic chemokine receptors are essential for stable cell adhesion and migration [66,67]. Here, we demonstrate that when chemokines and integrin ligands are copresented, their combined effect increases the adhesion of Tlymphocytes as compared to either molecule alone (Fig. 6). Future studies should investigate if ICAM-1 and HS-bound CXCL12 $\alpha$ promote cell adhesion independently, or if the enhanced adhesion is the consequence of any cooperative action involving cross-talk between receptors. For example, it has been proposed that the cellular signalling pathways triggered by CXCL12 $\alpha$ and ICAM-1 cooperate, increasing LFA-1 avidity to ICAM-1 [68]. Such a study will require analysis of cell signalling processes, which is amenable with our surfaces yet outside the scope of the current work.

The discussed examples illustrate that the strategy to create biomimetic surfaces described here represents a versatile experimental platform for mechanistic studies of GAG-protein interactions on the molecular and supramolecular scale, and of GAGmediated cell-cell and cell-matrix communication. The platform could also be useful for in vitro diagnostic studies and for drug development. Indeed the strategy could be used for the formation of surfaces presenting gradients of GAGs and proteins, to study the effect of specific compounds/drugs on T-lymphocyte migration during the immune response. Moreover, methods for the controlled presentation of chemokines by HS may be of key importance for the design of chemokine-loaded implantable devices for regenerative medicine or tissue remodelling purposes. For example, it was recently shown that CXCL12 $\alpha$-HS binding is necessary for post ischemia revascularization [69]. 


\section{Conclusions}

We have presented a versatile strategy to create biomimetic surfaces that present GAGs together with other cell surface or extracellular matrix molecules in a background of low non-specific binding. We have demonstrated that the orientation of the immobilized molecules can be controlled and their surface density tuned, thanks to the surface design and quantitative characterization by surface sensitive techniques, and how this platform can be used for functional studies on the molecular, supramolecular and cellular levels. T-lymphocytes adhere specifically to surfaces presenting CXCL12 $\alpha$ and CXCL12 $\alpha$ presented through HS enhanced cellular adhesion when co-immobilized with ICAM-1. The strategy to create multifunctional biomimetic surfaces should be broadly applicable for functional studies that require a well-defined supramolecular presentation of GAGs along with other matrix or cellsurface components.

\section{Acknowledgements}

We thank Nico Eisele and Luis Yate (both CIC biomaGUNE) for support with the SE measurements and for surface coatings, respectively, Damien Maurin (IBS) for producing recombinant CXCL12 $\alpha$, Rose-Laure Revel-Goyet, Françoise Lacroix and JeanPhilippe Kleman (IBS) for access to and support with the Microscope Platform, Nicole Thielens and Isabelle Bally (IBS) for access to and support from the SPR platform of the Partnership for Structural Biology in Grenoble, Jérôme Dejeu (DCM) for support with SPR experiments, and Pierre Labbé (DCM) for discussions. This work was supported by the Nanoscience Foundation Chair of Excellence Project "GAG2D", the NanoBio programme, the ICMG FR 2607, and LabEx ARCANE (ANR-11-LABX-0003-01).

\section{Appendix A. Supplementary data}

Supplementary data related to this article can be found online at http://dx.doi.org/10.1016/j.biomaterials.2014.07.017.

\section{References}

[1] Bernfield M, Götte M, Park PW, Reizes O, Fitzgerald ML, Lincecum J, et al. Functions of cell surface heparan sulfate proteoglycans. Annu Rev Biochem 1999;68:729-77.

[2] Cain SA, Baldock C, Gallagher J, Morgan A, Bax DV, Weiss AS, et al. Fibrillin-1 interactions with heparin. Implications for microfibril and elastic fiber assembly. J Biol Chem 2005;280:30526-37.

[3] Cain SA, Baldwin AK, Mahalingam Y, Raynal B, Jowitt TA, Shuttleworth CA et al. Heparan sulfate regulates fibrillin-1 $\mathrm{N}$ - and C-terminal interactions. J Biol Chem 2008;283:27017-27.

[4] Baranova NS, Nilebäck E, Haller FM, Briggs DC, Svedhem S, Day AJ, et al. The inflammation-associated protein TSG-6 cross-links hyaluronan via hyaluronan-induced TSG-6 oligomers. J Biol Chem 2011;286:25675-86.

[5] Day AJ, De la Motte CA. Hyaluronan cross-linking: a protective mechanism in inflammation? Trends Immunol 2005;26:637-43.

[6] Attili S, Richter RP. Self-assembly and elasticity of hierarchical proteoglycanhyaluronan brushes. Soft Matter 2013;9:10473-83.

[7] Lee GM, Johnstone B, Jacobson K, Caterson B. The dynamic structure of the pericellular matrix on living cells. J Cell Biol 1993;123:1899-907.

[8] Knudson W, Knudson CB. Assembly of a chondrocyte-like pericellular matrix on non-chondrogenic cells. Role of the cell surface hyaluronan receptors in the assembly of a pericellular matrix. J Cell Sci 1991;99(Pt 2):227-35.

[9] Laguri C, Sadir R, Rueda P, Baleux F, Gans P, Arenzana-Seisdedos F, et al. The novel CXCL12 $\gamma$ isoform encodes an unstructured cationic domain which regulates bioactivity and interaction with both glycosaminoglycans and CXCR4. PLoS One 2007;2:e1110.

[10] Johnson Z, Proudfoot AE, Handel TM. Interaction of chemokines and glycosaminoglycans: a new twist in the regulation of chemokine function with opportunities for therapeutic intervention. Cytokine Growth Factor Rev 2005;16:625-36.

[11] Duchesne L, Octeau V, Bearon RN, Beckett A, Prior IA, Lounis B, et al. Transport of fibroblast growth factor 2 in the pericellular matrix is controlled by the spatial distribution of its binding sites in heparan sulfate. PLoS Biol 2012;10: e1001361.

[12] Rueda P, Balabanian K, Lagane B, Staropoli I, Chow K, Levoye A, et al. The CXCL12gamma chemokine displays unprecedented structural and functional properties that make it a paradigm of chemoattractant proteins. PLoS One 2008; $3:$ e2543.

[13] Hesselgesser J, Liang M, Hoxie J, Greenberg M, Brass LF, Orsini MJ, et al. Identification and characterization of the CXCR4 chemokine receptor in human T cell lines: ligand binding, biological activity, and HIV-1 infectivity. J Immunol 1998;160:877-83.

[14] Weber M, Hauschild R, Schwarz J, Moussion C, De Vries I, Legler DF, et al. Interstitial dendritic cell guidance by haptotactic chemokine gradients. Science 2013;339:328-32.

[15] Altgärde N, Nilebäck E, De Battice L, Pashkuleva I, Reis RL, Becher J, et al. Probing the biofunctionality of biotinylated hyaluronan and chondroitin sulfate by hyaluronidase degradation and aggrecan interaction. Acta Biomater 2013;9:8158-66.

[16] Baranova NS, Foulcer SJ, Briggs DC, Tilakaratna V, Enghild JJ, Milner CM, et al. Inter- $\alpha$-inhibitor impairs TSG-6-induced hyaluronan cross-linking. J Biol Chem 2013;288:29642-53.

[17] Richter RP, Hock KK, Burkhartsmeyer J, Boehm H, Bingen P, Wang G, et al. Membrane-grafted hyaluronan films: a well-defined model system of glycoconjugate cell coats. J Am Chem Soc 2007;129:5306-7.

[18] Wolny PM, Banerji S, Gounou C, Brisson AR, Day AJ, Jackson DG, et al. Analysis of CD44-hyaluronan interactions in an artificial membrane system: insights into the distinct binding properties of high and low molecular weight hyaluronan. J Biol Chem 2010;285:30170-80.

[19] Reviakine I, Johannsmann D, Richter RP. Hearing what you cannot see and visualizing what you hear: interpreting quartz crystal microbalance data from solvated interfaces. Anal Chem 2011;83:8838-48.

[20] Richter R, Rodenhausen K, Eisele NB, Schubert M. Coupling spectroscopic ellipsometry and quartz crystal microbalance to study organic films at the solid-liquid interface. In: Hinrichs K, Eichhorn K-J, editors. Ellipsometry of functional organic surfaces and films. Berlin: Springer; 2014. pp. 223-48.

[21] Laguri C, Sapay N, Simorre J-P, Brutscher B, Imberty A, Gans P, et al. 13Clabeled heparan sulfate analogue as a tool to study protein/heparan sulfate interactions by NMR spectroscopy: application to the CXCL12 $\alpha$ chemokine. J Am Chem Soc 2011;133:9642-5.

[22] Bleul CC, Wu L, Hoxie JA, Springer TA, Mackay CR. The HIV coreceptors CXCR4 and CCR5 are differentially expressed and regulated on human T lymphocytes. Proc Natl Acad Sci U S A 1997;94:1925-30.

[23] Nagasawa T, Hirota S, Tachibana K, Takakura N, Nishikawa S, Kitamura Y, et al. Defects of B-cell lymphopoiesis and bone-marrow myelopoiesis in mice lacking the CXC chemokine PBSF/SDF-1. Nature 1996;382:635-8.

[24] Massena S, Christoffersson G, Hjertstrom E, Zcharia E, Vlodavsky I, Ausmees N, et al. A chemotactic gradient sequestered on endothelial heparan sulfate induces directional intraluminal crawling of neutrophils. Blood 2010;116: $1924-31$.

[25] Lortat-Jacob H. The molecular basis and functional implications of chemokine interactions with heparan sulphate. Curr Opin Struct Biol 2009;19:543-8.

[26] Mulloy B, Gee C, Wheeler SF, Wait R, Gray E, Barrowcliffe TW. Molecular weight measurements of low molecular weight heparins by gel permeation chromatography. Thromb Haemost 1997;77:668-74.

[27] Richter R, Mukhopadhyay A, Brisson A. Pathways of lipid vesicle deposition on solid surfaces: a combined QCM-D and AFM study. Biophys J 2003;85: 3035-47.

[28] Eisele NB, Frey S, Piehler J, Görlich D, Richter RP. Ultrathin nucleoporin phenylalanine-glycine repeat films and their interaction with nuclear transport receptors. EMBO Rep 2010;11:366-72.

[29] Domack A, Prucker O, Ruhe J, Johannsmann D. Swelling of a polymer brush probed with a quartz crystal resonator. Phys Rev E 1997;56:680-9.

[30] Johannsmann D, Reviakine I, Rojas E, Gallego M. Effect of sample heterogeneity on the interpretation of QCM(-D) data: comparison of combined quartz crystal microbalance/atomic force microscopy measurements with finite element method modeling. Anal Chem 2008;80:8891-9.

[31] D. Johannsmann. http://www2.pc.tu-clausthal.de/dj/software_en.shtml. n.d.

[32] Eisele NB, Andersson FI, Frey S, Richter RP. Viscoelasticity of thin biomolecular films: a case study on nucleoporin phenylalanine-glycine repeats grafted to a histidine-tag capturing QCM-D sensor. Biomacromolecules 2012;13:2322-32.

[33] Dubacheva GV, Curk T, Mognetti BM, Auzély-Velty R, Frenkel D, Richter RP. Superselective targeting using multivalent polymers. J Am Chem Soc 2014;136:1722-5.

[34] Mobley JL, Ennis E, Shimizu Y. Differential activation-dependent regulation of integrin function in cultured human T-leukemic cell lines. Blood 1994;83: 1039-50.

[35] Wolny PM, Spatz JP, Richter RP. On the adsorption behavior of biotin-binding proteins on gold and silica. Langmuir 2010;26:1029-34.

[36] Sarrazin S, Lamanna WC, Esko JD. Heparan sulfate proteoglycans. Cold Spring Harb Perspect Biol 2011;3:a004952.

[37] Richter RP, Brisson A. Characterization of lipid bilayers and protein assemblies supported on rough surfaces by atomic force microscopy. Langmuir 2003;19: $1632-40$

[38] Horton MR, Manley S, Arevalo SR, Lobkovsky AE, Gast AP. Crystalline protein domains and lipid bilayer vesicle shape transformations. J Phys Chem B 2007; 111:880-5. 
[39] Frey W, Schief WR, Vogel V. Two-dimensional crystallization of streptavidin studied by quantitative Brewster angle microscopy. Langmuir 1996;12: $1312-20$.

[40] Richter RP, Bérat R, Brisson AR. Formation of solid-supported lipid bilayers: an integrated view. Langmuir 2006;22:3497-505.

[41] Bingen P, Wang G, Steinmetz NF, Rodahl M, Richter RP. Solvation effects in the quartz crystal microbalance with dissipation monitoring response to biomolecular adsorption. A phenomenological approach. Anal Chem 2008;80: 8880-90.

[42] Hermens WT, Benes M, Richter R, Speijer H. Effects of flow on solute exchange between fluids and supported biosurfaces. Biotechnol Appl Biochem 2004;39: 277-84.

[43] Zhang Z, Xie J, Liu H, Liu J, Linhardt RJ. Quantification of heparan sulfate disaccharides using ion-pairing reversed-phase microflow high-performance liquid chromatography with electrospray ionization trap mass spectrometry. Anal Chem 2009;81:4349-55.

[44] Roussille L, Brotons G, Ballut L, Louarn G, Ausserré D, Ricard-Blum S. Surface characterization and efficiency of a matrix-free and flat carboxylated gold sensor chip for surface plasmon resonance (SPR). Anal Bioanal Chem 2011;401:1601-17.

[45] Fermas S, Gonnet F, Sutton A, Charnaux N, Mulloy B, Du Y, et al. Sulfated oligosaccharides (heparin and fucoidan) binding and dimerization of stromal cell-derived factor-1 (SDF-1/CXCL 12) are coupled as evidenced by affinity CEMS analysis. Glycobiology 2008;18:1054-64.

[46] Murphy JW, Cho Y, Sachpatzidis A, Fan C, Hodsdon ME, Lolis E. Structural and functional basis of CXCL12 (stromal cell-derived factor- $1 \alpha$ ) binding to heparin. J Biol Chem 2006;282:10018-27.

[47] Sadir R, Baleux F, Grosdidier A, Imberty A, Lortat-Jacob H. Characterization of the stromal cell-derived factor-1 $\alpha$-heparin complex. J Biol Chem 2001;276: $8288-96$.

[48] Soriano A, Martínez C, García F, Plana M, Palou E, Lejeune M, et al. Plasma stromal cell-derived factor (SDF)-1 levels, SDF1-3'A genotype, and expression of CXCR4 on T lymphocytes: their impact on resistance to human immunodeficiency virus type 1 infection and its progression. J Infect Dis 2002;186: 922-31.

[49] Ganju RK, Brubaker SA, Meyer J, Dutt P, Yang Y, Qin S, et al. The alphachemokine, stromal cell-derived factor-1alpha, binds to the transmembrane G-protein-coupled CXCR-4 receptor and activates multiple signal transduction pathways. J Biol Chem 1998;273:23169-75.

[50] Sadir R, Imberty A, Baleux F, Lortat-Jacob H. Heparan sulfate/heparin oligosaccharides protect stromal cell-derived factor-1 (SDF-1)/CXCL12 against proteolysis induced by CD26/dipeptidyl peptidase IV. J Biol Chem 2004;279: 43854-60.

[51] Hogg N, Laschinger M, Giles K, McDowall A. T-cell integrins: more than just sticking points. J Cell Sci 2003;116:4695-705.

[52] Yang Y, Jun CD, Liu JH, Zhang R, Joachimiak A, Springer TA, et al. Structura basis for dimerization of ICAM-1 on the cell surface. Mol Cell 2003;14: 269-76.
[53] Deisenhofer J. Crystallographic refinement and atomic models of a human Fc fragment and its complex with fragment B of protein A from Staphylococcus aureus at 2.9- and $2.8 \AA$ A resolution. Biochemistry 1981;20:2361-70.

[54] Semmrich M, Smith A, Feterowski C, Beer S, Engelhardt B, Busch DH, et al. Importance of integrin LFA-1 deactivation for the generation of immune responses. J Exp Med 2005;201:1987-98.

[55] Azcutia V, Routledge M, Williams MR, Newton G, Frazier WA, Manica A, et al. CD47 plays a critical role in T-cell recruitment by regulation of LFA-1 and VLA4 integrin adhesive functions. Mol Biol Cell 2013;24:3358-68.

[56] Reichel A, Schaible D, Al Furoukh N, Cohen M, Schreiber G, Piehler J. Noncovalent, site-specific biotinylation of histidine-tagged proteins. Anal Chem 2007;79:8590-600.

[57] Nisnevitch M, Firer MA. The solid phase in affinity chromatography: strategies for antibody attachment. J Biochem Biophys Methods 2001;49:467-80.

[58] Cha T, Guo A, Zhu X-Y. Enzymatic activity on a chip: the critical role of protein orientation. Proteomics 2005;5:416-9.

[59] Chevalier S, Cuestas-Ayllon C, Grazu V, Luna M, Feracci H, De la Fuente JM. Creating biomimetic surfaces through covalent and oriented binding of proteins. Langmuir 2010;26:14707-15.

[60] Murphy JW, Yuan H, Kong Y, Xiong Y, Lolis EJ. Heterologous quaternary structure of CXCL12 and its relationship to the CC chemokine family. Proteins 2009;78:1331-7.

[61] Schumann K, Lämmermann T, Bruckner M, Legler DF, Polleux J, Spatz JP, et al. Immobilized chemokine fields and soluble chemokine gradients cooperatively shape migration patterns of dendritic cells. Immunity 2010;32:703-13.

[62] Laguri C, Arenzana-Seisdedos F, Lortat-Jacob H. Relationships between glycosaminoglycan and receptor binding sites in chemokines-the CXCL12 example. Carbohydr Res 2008;343:2018-23.

[63] Ray P, Lewin SA, Mihalko LA, Lesher-Perez S-C, Takayama S, Luker KE, et al. Secreted CXCL12 (SDF-1) forms dimers under physiological conditions. Biochem J 2012;442:433-42.

[64] Ziarek JJ, Getschman AE, Butler SJ, Taleski D, Stephens B, Kufareva I, et al. Sulfopeptide probes of the CXCR4/CXCL12 interface reveal oligomer-specific contacts and chemokine allostery. ACS Chem Biol 2013;8:1955-63.

[65] Veldkamp CT, Seibert C, Peterson FC, De la Cruz NB, Haugner 3rd JC, Basnet H, et al. Structural basis of CXCR4 sulfotyrosine recognition by the chemokine SDF-1/CXCL12. Sci Signal 2008;1:ra4.

[66] Bargatze RF, Butcher EC. Rapid G protein-regulated activation event involved in lymphocyte binding to high endothelial venules. J Exp Med 1993;178:367-72.

[67] Von Andrian UH, Mackay CR. T-cell function and migration. Two sides of the same coin. N Engl J Med 2000:343:1020-34.

[68] Weber KS, Ostermann G, Zernecke A, Schröder A, Klickstein LB, Weber C. Dual role of H-Ras in regulation of lymphocyte function antigen- 1 activity by stromal cell-derived factor-1alpha: implications for leukocyte transmigration. Mol Biol Cell 2001;12:3074-86.

[69] Rueda P, Richart A, Récalde A, Gasse P, Vilar J, Guérin C, et al. Homeostatic and tissue reparation defaults in mice carrying selective genetic invalidation of CXCL12/proteoglycan interactions. Circulation 2012;126:1882-95. 